Published in final edited form as:

Nat Neurosci. 2018 October 01; 21(10): 1412-1420. doi:10.1038/s41593-018-0225-y.

\title{
Restoring wild type-like CA1 network dynamics and behaviour during adulthood in a mouse model of schizophrenia
}

\author{
Thomas Marissal ${ }^{\# 1,4,{ }^{*}, \text { Rodrigo F. Salazar }}{ }^{\# 1}$, Cristina Bertollini ${ }^{\# 1}$, Sophie Mutel ${ }^{1,2}$, Mathias \\ De Roo ${ }^{1,3}$, Ivan Rodriguez ${ }^{2}$, Dominique Müller ${ }^{1, \dagger}$, Alan Carleton ${ }^{1, *}$ \\ ${ }^{1}$ Department of Basic Neuroscience, Faculty of Medicine, University of Geneva, Geneva, \\ Switzerland ${ }^{2}$ Department of Genetics and Evolution, Faculty of Sciences, University of Geneva, \\ Geneva, Switzerland ${ }^{3}$ Department of Anesthesiology, Pharmacology and Intensive Care, \\ University Hospital of Geneva, Geneva, Switzerland \\ \# These authors contributed equally to this work.
}

\begin{abstract}
Schizophrenia is a severely debilitating neurodevelopmental disorder. Establishing a causal link between circuit dysfunction and particular behavioural traits relevant to schizophrenia is crucial to shed new light on the mechanisms underlying the pathology. Here we studied an animal model of the 22q11 deletion syndrome, which represents the highest genetic risk to develop the pathology. We report a desynchronization of hippocampal neuronal assemblies that results from parvalbumin interneuron hypoexcitability. Rescuing parvalbumin interneuron excitability with pharmacological or chemogenetic approaches is sufficient to restore wild type-like CA1 network dynamics and hippocampal-dependent behaviour during adulthood. In conclusion, our data provide some insights underlying network dysfunction relevant to schizophrenia and highlight the use of reverse engineering in restoring physiological and behavioural phenotypes in an animal model of neurodevelopmental disorder.
\end{abstract}

\footnotetext{
*Correspondence should be addressed to A.C. (alan.carleton@unige.ch) or T.M. (thomas.marissal@inserm.fr).

${ }_{4}^{4}$ Present address: Institut de Neurobiologie de la Méditerranée, UMR 1249 INSERM, Marseille, France

$\dagger$ Deceased on 29 April 2015.

Data availability. The data that support the findings of this study are available from the corresponding authors upon reasonable request

Code availability. The custom-written MATLAB scripts are available from the corresponding authors upon reasonable request. Author Contributions

T.M., D.M. and A.C. carried out the study conceptualization. T.M., R.S., C.B., S.M., M.D.R., I.R., D.M. and A.C. contributed to the experimental design. TM performed the calcium imaging experiments and their analyses. C.B. carried out the in vitro electrophysiological experiments. R.S. performed the in vivo electrophysiological recordings and developed most of the MATLABbased programs used for the analysis of the calcium imaging and the electrophysiological recordings. M.D.R. developed some MATLAB-based scripts used to analyse calcium-imaging data. T.M. and C.B. performed stereotaxic viral infections. T.M. performed behavioural experiments with the help of S.M. A.C., T.M., R.S. and I.R. wrote and edited the manuscript with comments from all other authors.
}

Competing Financial Interests Statement

The authors declare no competing financial interests. 


\section{Keywords}

ensemble activity; parvalbumin interneuron; synchronization; schizophrenia; 22q11 deletion syndrome

\section{Introduction}

Alterations of network dynamics have been proposed to be instrumental in schizophrenia ${ }^{1-3}$. Yet it remains unclear how a neurodevelopmental circuit dysfunction is caused by altered neuronal physiology and whether such a dysfunction could be corrected during adulthood.

A specific population of inhibitory neurons, the Parvalbumin interneurons (PVIs), plays a key role in regulating network dynamics ${ }^{4-7}$ and a growing body of evidence in rodents suggests that they play a causal role in neuronal dysfunctions relevant to schizophrenia ${ }^{8-11}$. In line, mouse models in which PVI properties are modified can reproduce some behavioural phenotypes relevant to schizophrenia ${ }^{7,12-16}$. However, it remains unclear whether manipulating specifically PVIs during adulthood could restore altered physiology and behaviour in animal models associated to a genetic risk factor identified in humans.

Among various genetic alterations, the specific deletion of $\sim 30$ genes on chromosome 22 that leads to the 22q11 deletion syndrome (22q11DS), is the highest identified genetic risk to develop schizophrenia ${ }^{17-19}$. We used a genetically engineered mouse bearing a hemizygous deletion on chromosome 16, termed Lgdel/+, which replicates the chromosomal alteration of the human 22q11DS ${ }^{18}$. In the CA1 area of the hippocampus, mouse models of 22q11DS differ from wild-type (WT) animals regarding their structural ${ }^{20-22}$ and electrophysiological properties ${ }^{23}$, and their functional connectivity with distant brain areas ${ }^{3}$. In the present study, we report alterations in network dynamics and behaviour in the Lgdel/+ model of the 22q11DS and we developed a rescue strategy based on PVI manipulations during adulthood.

\section{Results}

\section{Hyposynchronization of pyramidal cells in the Lgdel/+ CA1 hippocampal network in vitro}

Neural activity was monitored in hippocampal slice cultures using the genetically encoded calcium indicator GCaMP6s expressed by CA1 neurons following adeno-associated viral (AAV) vector transfection (Fig. 1a,b). Network dynamics were induced by bath application of carbachol (see methods), which triggered spontaneous calcium activity in individual neurons of wild type (WT, +/+) mice (Fig. 1c,d, Supplementary Fig. 1 and Supplementary Video 1). Likewise, individual CA1 neurons of Lgdel/+ mice exhibited spontaneous calcium activity during the duration of the recording. Neither the fraction of active neurons (Fig. 1e), the mean frequency (Fig. 1f), the mean amplitude (Fig. 1g) nor the mean duration (Fig. 1h) of calcium transients was significantly different between genotypes. In contrast, CA1 ensemble dynamics was altered in Lgdel/+ mice (Supplementary Video 2). Indeed, neurons were desynchronized in respect to each other and the oscillations observed at a population level were reduced in Lgdel/+ mice (Fig. 1c,d). We thus quantified population activity and neuronal correlations and compared these quantities to data randomly shuffled in time (see 
methods). This enabled to control for the influence of neuronal co-activation occurring by chance (Supplementary Fig. 2 and 3). Lgdel/+ mice displayed less co-active cells (Fig. 1i), fewer occurrences of these co-activations (Fig. 1j) and less correlated neurons (Fig. 1k,l) with respect to WT animals. In summary, Lgdel/+ slices exhibited a desynchronization of the CA1 hippocampal network.

\section{PVI hypoexcitability and pyramidal neuron hypoinhibition in the CA1 area of Lgdel/+ mice}

To gain further insights into the mechanism underlying altered CA1 dynamics, we performed patch-clamp recordings of various neuronal populations. First, we recorded spontaneous excitatory and inhibitory post-synaptic currents (sEPSCs and sIPSCs, respectively) in pyramidal cells (Fig. 2a). Consistent with a previous report ${ }^{23}$, CA1 pyramids recorded from Lgdel/+ mice had a comparable number of sEPSCs but less sIPSCs per second compared to CA1 pyramids in WT mice (Fig. 2a-d). In addition, no differences in sPSC amplitude (Supplementary Fig. 4a-c), in mEPSC frequency (Supplementary Fig. 4d) and in mIPSC frequency (Fig. 2e) were observed between the two genotypes. These results provide evidence that hippocampal pyramids in Lgdel/+ mice are characterized by hypoinhibition, likely to originate from the alteration of the firing activity of GABAergic neurons.

We further tested the role of PVI neurons in the hypoinhibition of pyramidal neurons by recording genetically labelled PVIs (Fig. 2f-n). An analysis of input/output function in current-clamp experiments revealed that CA1 PVIs were less excitable in Lgdel/+ than in WT animals when recorded in artificial cerebrospinal fluid (Fig. 2g,h). Interestingly, such firing difference disappeared when $\mathrm{GABA}_{\mathrm{A}}$, AMPA and NMDA receptors were blocked pharmacologically (Fig. 2i). Thus, PVI hypoexcitability may rather reflect differences in PVI synaptic inputs than changes of their intrinsic properties. Supporting this hypothesis, CA1 PVIs recorded from Lgdel/+ mice received similar number of sEPSCs but more sIPSCs per second than CA1 PVI neurons recorded in WT animals (Fig. 2j,k). No difference in sPSC amplitude (Supplementary Fig. 4f-g), mEPSC frequency (Supplementary Fig. 4h) or mIPSC frequency (Fig. 2n) was observed. Taken together, our data show that CA1 pyramidal cells of Lgdel/+ mice are hypoinhibited and that PVIs are hypoexcitable.

\section{Increasing PVI excitability restores wild-type like CA1 network dynamics in Lgdel/+ mice}

Could altered network dynamics observed in slices be induced by PVI hypoexcitability? To address this question, we used a cell-autonomous chemogenetic approach by specifically infecting CA1 PVIs from $P$ valb $b^{\text {cre/+} ;+/+~ m i c e ~ w i t h ~ A A V ~ e n a b l i n g ~ t h e ~ c o n d i t i o n a l ~ e x p r e s s i o n ~}$ of the inhibitory designer receptor exclusively activated by designer drug (DREADD) hM4Gi, which is selectively activated by the drug clozapine-N-oxide (CNO, see methods). Reducing PVI excitability desynchronized CA1 assemblies in WT slices (Fig. 3a-e).

Conversely, could hippocampal network dynamics be rescued in Lgdel/+ mice by increasing PVI excitability? First, we used a pharmacological approach by using a Neuregulin 1 peptide (NRG1P), which increases PVI excitability in WT mice ${ }^{24}$ without altering network dynamics or behaviour in WT mice (Supplementary Fig. 5). Second, we specifically expressed the excitatory DREADD hM3Gq in CA1 PVIs from $P_{v a l l} b^{\text {cre/+}} ;$ Lgdel/+ mice. 
During patch-clamp recordings of hippocampal cultured slices, both strategies raised PVI excitability and restored input/output functions comparable to the ones recorded from $P_{\text {valb }}$ cre/++$_{++/+}$mice (Fig. 4a-f; difference between $P$ valb $b^{\text {cre/++} ;+/+~ a n d ~ t r e a t m e n t s ~ i n ~}$ $P_{\text {val }} b^{\text {cre/+ }} ;$ Lgdel/ + mice: two-way repeated measures ANOVA $F_{1,29}=0.44, P=0.51$ and $F_{1,28}=0.12, P=0.73$ for NRG1P and CNO treatment, respectively). In line with the patchclamp data, PVIs from $P_{V a l} b^{\text {cre/+}} ;$ Lgdel/+ mice generate less calcium transients than PVIs from $P_{\text {val }} b^{\text {cre/+}} ;+/+$ mice during slice imaging after carbachol application. Similarly, the frequency of the calcium events recorded in PVIs from $P_{\text {val }} b^{\text {cre/+}} ;$ Lgdel/+ cultured slices increased during the application of NRG1 or CNO and restored frequencies comparable to the ones recorded from $P$ valb ${ }^{\text {cre/+}} ;+/+$ mice (Fig. 4g,j; two-sided Mann-Whitney test $\mathrm{U}=$ 241 and 248, $P=0.77$ and 0.63 for NRG1P and CNO treatment, respectively).

We then tested the effect of the two treatments on CA1 network dynamics by pre-incubating hippocampal cultured slices with either NRG1P or CNO prior to calcium imaging. Strikingly, both strategies increased neuronal correlations (Fig. 5a-d) and co-activations (Supplementary Fig. 6e-f) in Lgdel/+ mice to a level comparable to WT littermates (Fig. 5c, Two-Sided Mann-Whitney test $\mathrm{U}=90$ and 74, $P=0.73$ and 0.89 for NRG1P and CNO treatment, respectively). Thus, counterbalancing the PVI hypoexcitability of Lgdel/+ mice was sufficient to extinguish the network desynchronization observed in brain slices.

Although 22q11DS is considered to be a neurodevelopmental disorder, we investigated whether the pharmacological and chemogenetic treatments used above could also be efficient in adult animals. We first performed in vivo electrophysiological recordings in the dorsal CA1 area (dCA1) of awake mice (Fig. 6a and Supplementary Fig. 7a). A spectral analysis of the local field potential (LFP) revealed lower power in Lgdel/+ mice compared to WT animals in the theta frequency band (5-8Hz; Fig. 6a,b and Supplementary Fig. 7b). Neuronal oscillations in the theta frequency band are important to hippocampal functions and their strength can be modulated by PVI activations ${ }^{5}$. Interestingly, NRG1P injections significantly increased the power of theta oscillations in Lgdel/+ but not in WT mice (Fig. 6a-c and Supplementary Fig. 5e,f, respectively). To improve the specificity of our manipulation, we infected dCA1 PVI of $P_{\text {val }} b^{\text {cre/+ }} ;$ Lgdel/+ mice with an AAV enabling the conditional expression of the hM3Gq DREADD (Supplementary Fig. 7c). CNO injections also significantly elevated the LFP theta power (Fig. 6a-c). Interestingly, Lgdel/+ mice treated with NRG1P or CNO during 60 min no longer display any significant difference with the "control" mice (i.e. WT 60 min after I.P. injection of NRG1P; Two-Sided Mann-Whitney test; $P=0.65$ and 0.48 , respectively). In order to determine if these results were specific to the theta frequency band, we performed a similar analysis on the delta $(1-4 \mathrm{~Hz})$ and the gamma $(30-80 \mathrm{~Hz})$ frequency bands. The power of oscillations in the delta frequency band did not differ between WT and Lgdel/+ animals (Supplementary Fig. 8a). In contrast, the power measured in the gamma frequency range was lower in Lgdel/+ compared to WT mice (Supplementary Fig. 8b). When pharmacological and cell-autonomous pharmacogenetic treatments were applied, the power in the gamma frequency range increased (Supplementary Fig. 8c).

As LFP analyses reveal the neural dynamics occurring at a mesoscale level, we wanted to know if these effects were also observable at a finer scale. During the same recordings, we 
isolated single units and quantified their cross-correlation (see methods). As previously reported ${ }^{25}$, the majority of the correlations occurred around zero-phase lag $( \pm 20 \mathrm{~ms}$; Fig. $6 \mathrm{~d})$. To compare the different treatments, the highest fraction of correlated neurons among all available lags was evaluated for each recording. Consistent with our in vitro findings, Lgdel/+ mice had less correlated pairs than their WT littermates (Fig. 6e). When NRG1P was administered to $\mathrm{Lgdel} /+^{-}$mice or $\mathrm{CNO}$ injected to $P v a l b^{\text {cre/+}} ; \mathrm{Lgdel} /+$ mice, the fraction of correlated cells increased consistently (Fig. 6f). In summary, NRG1P injection and chemogenetic excitation of PVIs in adult animals increased the occurrence of neuronal correlations and the power of theta oscillations in awake Lgdel/+ mice.

\section{Increasing PVI excitability restores hippocampal-dependent behavioural deficits in Lgdel/ + mice}

Could the same pharmacological and chemogenetic manipulations also restore WT-like behaviours? As observed in various animal models of schizophrenia ${ }^{26}$, including the 22q11DS mouse model ${ }^{21}$, adult Lgdel/+ mice exhibit hyper-locomotor activity in comparison to their WT littermates in the open field test (OFT). Intra-peritoneal injections of NRG1P in Lgdel/+ mice (30-60 min prior to the testing) and selective excitation of dCA1 PVI expressing the hM3D DREADD led to a reduction in the distance travelled in the OFT to levels similar to the ones reached by WT littermates (Two-Sided Mann-Whitney test $P=$ $0.15(\mathrm{U}=93)$ and $P=0.61(\mathrm{U}=57)$, respectively; Fig. 7a,b and Supplementary Fig. 5i,j). Next, we measured the acoustic startle reflex and its pre-pulse inhibition (PPI), which are considered as relevant biomarkers of schizophrenia ${ }^{27}$. As previously reported ${ }^{21,28}$, we observed that Lgdel/+ mice exhibited both a higher startle response and a lower PPI compared to WT mice. When either CNO or NRG1P were injected, the startle response in Lgdel/+ mice was decreased to a level comparable to the one observed in WT animals (Supplementary Fig. 9a,b). Moreover, when NRG1P was administrated intraperitoneally, the PPI deficit of Lgdel/+ mice was also rescued (Supplementary Fig. 9c). In contrast, such a rescue did not occur when the dCA1 PVI excitability was enhanced chemogenetically. This result is consistent with the notion that hippocampal processing is not necessary to PPI ${ }^{29}$. Finally, to assess memory deficits relevant to schizophrenia ${ }^{26,30}$, we used contextual fear conditioning (Fig. 7c). As previously reported for other 22q11DS mouse models 21,31, Lgdel/+ mice displayed a deficit in fear memory response compared to WT mice (Fig. 7d). The latter difference cannot be explained by a general tendency of Lgdel/+ mice to freeze less, as we observed no genotype difference in the freezing behaviour during the conditioning (Supplementary Fig. 9d). During contextual fear conditioning, the injection of NRG1P and the activation of hM3Gq increased the conditional response of $\mathrm{Lgdel} /+$ mice to WT levels (comparison with WT values: Two-Sided Mann-Whitney test $P=0.69(\mathrm{U}=72)$ and $0.28(\mathrm{U}=57)$ for NRG1P and CNO treatment, respectively) and thereby improved their hippocampal-dependent memory (Fig. 7d,e and Supplementary Fig. 5k). In conclusion, increasing the excitability of PVI during adulthood is not only sufficient to restore CA1 network dynamics but also to restore wild-type like behaviour in Lgdel/+ mice. 


\section{Discussion}

In the present study, we tested the causal relationship between deficits at a synaptic/ physiological deficiencies, anomalies in network dynamics and behaviour using an animal model of the 22q11DS. Although both cortical ${ }^{32}$ and the hippocampal networks ${ }^{33}$ may be involved in the pathophysiology of schizophrenia and of the 22q11DS, we focused our work on the latter area. Effectively, hippocampi originating from mouse models of the disorders display alterations in the properties of the synaptic and neuronal components ${ }^{20-23}$, as well as the circuit dynamics ${ }^{3,34}$. Our in vitro hippocampal preparation enabled us to identify a deficit in CA1 ensemble dynamics (Fig. 1), which is causally linked to the reduced excitability of PVIs (Figs. 2,3 and 5). It also enabled us to develop a rescue strategy based on the increase of PVI excitability using either a PVI-specific chemogenetic approach or a pharmacological strategy (i.e. Neuregulin 1 peptide; Figs. 4 and 5). Importantly, the rescue strategies were further validated in vivo in adult animals. We report a deficit in the synchronized response of the CA1 neuronal network in awake Lgdel/+ mice, reflected by a diminished spike correlation between neuronal pairs and a and a reduction of neuronal oscillations in the theta frequency range (Fig. 6 and Supplementary Fig. 8). These deficits were either diminished or abolished by manipulations of PVI excitability.

We also manipulated PVI excitability during various behaviours, which involve hippocampal processing and are relevant to the intellectual disabilities associated with the 22q11DS 18 (Fig. 7). Using the Df16/+ mouse model of 22q11DS, Stark and colleagues observed differences with wild-type animals in an open-field exploration, during contextual fear conditioning and during the pre-pulse inhibition (PPI) of the acoustic startle reflex ${ }^{21}$. Consistently, we found differences between Lgdel/+ mice and wild-type littermates. We eliminated these alterations in the open-field and during contextual fear-conditioning by modulating the excitability of dCA1 PVI using a specific chemogenetic manipulation. The same manipulation improved the impaired startle reflex but failed to restore the PPI deficit observed in Lgdel/+ mice. In contrast, the systemic injection of NRG1P, probably affecting various brain regions, rescued the PPI deficit. As hippocampal processing is not necessary to PPI ${ }^{29}$, the latest results suggest that the recovery of PPI by NRG1P administration is operating through other brain regions than the dorsal hippocampus. In line with the latter assumption, it was recently reported that the schizophrenia-related circuit dysfunction and behavioural alterations observed in adult Dlx $5 / 6^{+/-}$mice are rescued by optogenetic activation of prefrontal cortex interneurons. However, PVI-specific manipulations were not specifically tested ${ }^{7}$.

In the present study, we made no attempt to dissect the molecular mechanisms underlying the cellular and network alterations of the Lgdel/+ mouse model. Testing the causal relationship between every of the $\sim 30$ genes deleted in this pathology 18 and the neuronal/ circuit properties would represent a long task with uncertain outcomes. Although NRG1 and its receptor Erbb4, on which we based a rescue strategy, are associated with schizophrenia in both rodents ${ }^{13}$ and in humans ${ }^{35}$, none of the genes of its molecular pathway seems to be comprised in the 22q11DS deletion. Other groups have identified an alteration of the ZDHHC8-Gsk3 pathway, which could be a neuronal substrate to the network and behavioural deficits related to the 22q11DS 36,37 . However, the rescue strategies targeting 
the ZDHHC8-Gsk3 are efficient only during a narrow period of development and are not likely to restore the behavioural phenotypes during adulthood.

PVI dysfunction has also been observed in other animal models related to schizophrenia 7, 10, 12-16. Therefore, we hypothesize that different molecular mechanisms could lead to identical alterations of PVI properties, which could be restored using a similar strategy. In that case, PVI neurons would represent a possible therapeutical target for various diseases regardless their molecular origin. Determining the impact of PVI modulation on other genetic or drug-induced models of neuropsychiatric diseases will need further investigations. In addition, it remains unclear whether the impaired PVIs belong to a specific subclass of Parvalbumin-expressing interneurons ${ }^{38-42}$. It is well accepted that there are different subtypes of CA1 PVIs, among which basket cells are the most abundant ${ }^{38}$ and count for more than $70 \%$ in the CA1 stratum pyramidale ${ }^{39}$. To reduce the variability related to the heterogeneity of PVIs, we limited the in vitro characterisation of the synaptic and electrophysiological properties of the CA1 PVI to the pyramidal layer, which may include bi-stratified ${ }^{40}$ and axo-axonic cells ${ }^{43}$; two cell types related to schizophrenia 44,45 . Since our chemogenetic rescue strategy did not discriminate between different PVI subtypes, more specific genetic manipulations will be required to test the relative contributions of various PVI subpopulations.

In line with the previous point, we made no attempt to identify the neuronal source of the increased inhibition received by PVIs located in the CA1 pyramidal cell layer of Lgdel/+ slices. This hyper-inhibition might appear paradoxical given that PVIs, which are known to inhibit each other in the hippocampus ${ }^{46}$, are hypo-excitable in the mutants. However, we cannot exclude the possibility that PVIs located outside of the pyramidal cell layer, that we have not recorded, might be hyper-excitable in Lgdel/+ mice. Alternatively, PVIs might also receive inhibitory inputs from other classes of Parvalbumin-negative interneurons ${ }^{47,48}$, presumably hyper-excitable in Lgdel/+ mice. A recent study, which used single cell RNA sequencing, suggests the existence of potentially $\sim 50$ subclasses of GABAergic interneurons in the CA1 region of the mouse hippocampus alone ${ }^{49}$ (which includes nine different clusters of PVIs). Therefore, to characterize the different classes of PVI-inhibiting interneurons and to identify the ones that are hyper-excitable in Lgdel/+ mice is an entire study on its own and should be the purpose of future research.

In conclusion, our findings provide mechanistic insights into the alterations observed at a network level in an animal model related to a human genetic disease, the 22q11DS. Our work suggests that the hypo-excitability of subpopulations of inhibitory neurons (e.g. PVIs) leads to an alteration of the neuronal synchronization in the hippocampus and probably in other brain areas ${ }^{50}$. Although the 22q11DS is a neurodevelopmental disorder, our data demonstrate that selective neuronal manipulations during adulthood are sufficient to restore functional brain dynamics and typical behavioural patterns. Furthermore, our results highlight PVIs as a valuable therapeutical target for 22q11DS and similar neuropsychiatric disorders. 


\section{Online Methods}

\section{Animals}

We used mice of both sexes up to 10 months (i.e. at the end of the experiments). Animals were maintained on a normal $12 \mathrm{~h} \mathrm{light/dark} \mathrm{cycle} \mathrm{at} 24^{\circ} \mathrm{C}$ with ad libitum access to food and water. We crossed Lgdel/+ (Del(16Dgcr2-Hira)1Rak ${ }^{51}$, generously provided by Prof. Raju Kucherlapati, Harvard university), characterized by a deletion of the 22q11DS-related genes, with C57BL/6J wild-type mice (Janvier, France) to generate Lgdel/+ mice and wild-type (WT) littermates. Homozygous $P_{v a l} b^{\text {cre/cre }}$ mice $\left(P_{v a l b} b^{\text {tm1 (cre)Arbr/J }}\left[{ }^{52}\right]\right.$, Jackson laboratory, \#017320; C57BL/6J background) were crossed with Lgdel/+ mice to generate $P$ valb ${ }^{\text {cre/+} ;+/+~}$ and $P$ val $b^{\text {cre/+}} ;$ Lgdel/ + littermates. Animal assignment to the various experimental groups was randomized between the littermates within each cage. Thus, each cage contained all the treatments. The genotype of the animals was unknown to the experimenters during the experiments.

All animal protocols are in accordance with the Swiss Federal Act on Animal Protection, with the Swiss Animal Protection Ordinance and were approved by the University of Geneva and Geneva state ethics committees (authorization numbers: 1007/3129/0 and GE/156/14).

\section{Chemical reagents}

Carbachol (CCh) was purchased from Sigma-Aldrich. Clozapine-N-oxide (CNO) was purchased from Enzo Life Sciences. A recombinant polypeptide containing the extracellular domain of beta1 Neuregulin 1 (NRG1P), able to cross over the brain blood barrier ${ }^{53,54}$, was purchased from Propsec. Tetrodotoxin (TTX) was purchased from Latoxan. D-2-amino-5phosphonopentanoic acid (D-APV), 6,7-dinitroquinoxaline-2,3-dione (DNQX), and Gabazine (GBZ) were purchased from Abcam Biochemicals.

\section{Adeno-associated viruses}

Adeno-associated viruses (AAVs) were produced by the University of North Carolina and the University of Pennsylvania vector cores. Calcium signals were detected using a recombinant AAV1.hSyn.GCaMP6s.WPRE.SV40, which enables the expression of the calcium-sensor GCaMP6s in neurons. The recombinant AAV5-hSyn-DIO-hM4D(Gi)mCherry and AAV5-hSyn-DIO-hM3D(Gq)-mCherry 55 construct were used for chemogenetic inhibition and excitation of Cre-expressing PVIs, respectively. AAV9.CAG.Flex.tdTomato.WPRE.bGH was used in order to specifically label Creexpressing PVIs with a red fluorophore.

\section{Slice preparation}

Five to six day-old pups were decapitated and their hippocampi were carefully removed and unfolded. Transverse hippocampal slices ( $400 \mu \mathrm{m}$ thickness) were made using a chopper (McIlwain). Slices were then maintained in an incubator at $33^{\circ} \mathrm{C}$ in a culture medium as previously described ${ }^{56}$. Viral infection of the slices was performed four days after the preparation of the culture (day in vitro 4, DIV4), by putting a hydrophilic membrane (FHLC white membrane, Millipore) on the slice surface. A drop of AAV-containing solution (approximately $0.1 \mathrm{~mL}$ ) was placed on the surface of the targeted CA1 region of the 
hippocampus using a pressurized glass pipette (Harvard Apparatus and Toohey Spritzer, Toohey Company). The membrane was left on the slice for $1 \mathrm{~h}$ in an incubator at $33^{\circ} \mathrm{C}$ before being removed. Calcium imaging and patch-clamp experiments were conducted around one week after the viral infection that is from DIV11 onwards.

\section{Calcium imaging}

Slices previously infected at DIV4 with an AAV virus leading to the expression of the genetically encoded calcium sensor GCaMP6s ${ }^{57}$ under the control of the human synapsin promoter were used. In some experiments, AAV enabling the conditional expression of the excitatory DREADD hM3Gq ${ }^{58}$ or the red fluorophore tdTomato were also injected. Slices were immersed in an artificial cerebro-spinal fluid (ACSF) containing (in $\mathrm{mM}$ ): $\mathrm{NaCl}$ (124), $\mathrm{KCl}$ (1.6), $\mathrm{KH}_{2} \mathrm{PO}_{4}$ (1.2), $\mathrm{MgCl}_{2}$ (1.3), $\mathrm{CaCl}_{2}$ (2) Glucose (10), and ascorbic acid (2.0), $\mathrm{pH}$ 7.4 , continuously oxygenated and perfused at room temperature $\left(\sim 22^{\circ} \mathrm{C}\right)$ using a peristaltic pump. Calcium transients were recorded using a Nipkow-type spinning disk confocal microscope (Olympus) coupled with single-photon lasers (excitation wavelength 488 and $565 \mathrm{~nm}$ ). Images were acquired through a CCD camera (Visitron Systems Evolve). Slices were imaged using a 10X 0.30NA objective (Olympus) at $8.9 \mathrm{~Hz}$ frame rate (i.e. $112 \mathrm{~ms}$ per frame). A few calcium-imaging experiments ( $n=5 \mathrm{WT}$ and $9 \mathrm{Lgdel} /+$ slices) were performed using a multibeam two-photon laser scanning system (same frame rate as above, Trimscope, LaVision Biotec, Germany) mounted on a BX51WI Olympus microscope coupled to Ultra II chameleon laser (Coherent). A typical imaging session covered a field of $420 x 420 \mu \mathrm{m}$ size containing 250 individual neurons in the CA1 area. Network dynamics were induced by bath application of carbachol ${ }^{59,60}$. Slices were recorded for 6 minutes either before or five minutes after carbachol $(\mathrm{CCh})$ application. For some experiments, slices were pre-incubated with NRG1P $(10 \mathrm{nM})$ or $\mathrm{CNO}(1 \mu \mathrm{M}) 15$ minutes prior to CCh application. All drugs remained present during the entire recordings.

\section{Analyses of the calcium imaging data}

Calcium data analyses ${ }^{61}$ were performed using the Matlab-based 'Caltracer3beta' software (Columbia University), which enables a) the tracing of Regions Of Interests (ROIs) matching the identified GCaMP6s-expressing neuronal soma, b) the calculation of the averaged fluorescence signal from each cell-based ROIs as a function of time. In order to distinguish the actual neuronal calcium activity from background activity (i.e. fibres nearby cells), the fluorescence within a halo of the pixels surrounding the ROI was subtracted from the fluorescence signal recorded in the ROI. From the resulting signal, the onsets and offsets of the calcium events were identified. Onsets were automatically detected when fluorescence exceeded a threshold set for every slice (based on the background noise) during a minimum time of 1s (based on the kinetics of GCaMP6s), and offsets were defined as the events halfdecay time. Onsets and offsets were manually corrected after automatic detection, and used to estimate 1) the proportion of active neurons and the frequency of calcium events, 2) the amplitude and the duration of calcium events, 3) the proportion of co-activated neurons and the duration of their co-activation and 4) the proportion of correlated pairs of neurons and their preferred lags. These analyses were performed using custom-made Matlab-based scripts. 
Calcium event amplitude was calculated considering the cell fluorescence value at the onset of the event (F0) and the maximum value of fluorescence measured during the event (F), and was scored as (F-F0)/F0 in \%. All signals below 5\% were considered as noise. Calcium event duration was calculated by subtracting the offset to the onset time values. An average event amplitude and duration was calculated for each neuron in each experiment (point 2 above).

The co-activation of neurons (point 3 above) is the number of onsets over the whole population for each frame. This co-activation reached significance if its value was above the maximum number of onsets found in a surrogate population obtained by randomization. The randomization (10000 iterations) consisted in adding a random shift (between one and the total number of frames minus one) to the onsets of each neuron; each neuron had a different shift assigned. When the shifted onsets exceeded the last frame, the difference was referenced to the first frame. This procedure maintained the same activity pattern for each neuron but scrambled the relationship between neurons. This method provided an estimate of the fraction of co-active neurons that exceeded chance levels and the fraction of frames when it occurred. Note that our method is very conservative and tends to underestimate the actual number of co-active neurons especially in synchronized populations.

The correlation between two neurons (point 4 above) was quantified as the number of onset coincidences occurring between lags spanning -20 to +20 frames and binned into two frames. Significant correlation between two neurons occurred when the number of coincidences exceeded a chance threshold at any lag. The threshold was obtained as the maximum number of coincidences found in a surrogate population (2000 iterations) generated by a similar randomization procedure as described in the previous paragraph. The only difference was that the random shift occurred within the range of the lags used with the exclusion of zero-lag. This procedure highlights pairs of neurons that are co-active within 20 frames and that have preferred lags, usually only one, above chance.

\section{Patch-clamp electrophysiology}

Recordings were done from slices perfused with oxygenated ACSF containing (in $\mathrm{mM}$ ): $\mathrm{NaCl}$ (124), $\mathrm{KCl}$ (1.6), $\mathrm{NaHCO}_{3}$ (24), $\mathrm{KH}_{2} \mathrm{PO}_{4}$ (1.2), $\mathrm{MgCl}_{2}$ (1.3), $\mathrm{CaCl}_{2}$ (2) Glucose (10), and ascorbic acid (2.0), $\mathrm{pH} 7.4$, continuously oxygenated and perfused at room temperature $\left(\sim 22^{\circ} \mathrm{C}\right)$ using a peristaltic pump. CA1 pyramidal cells were visually identified using infrared video microscopy. PVIs were identified by fluorescence after the infection of $P_{\text {val }}$ cre/++$_{++/+}$and $P_{\text {val }}{ }^{\text {cre/+}} ;$ Lgdel//+ slices with AAVs (see above). We specifically recorded PVIs localized in the pyramidal layer, which are in majority basket cells ${ }^{39}$. For recordings in current clamp mode, cells were recorded using patch-clamp pipettes (tip resistance, 3-5 M $\Omega$ ) filled with an intracellular solution containing (in $\mathrm{mM}$ ) $70 \mathrm{~K}$-gluconate, $70 \mathrm{KCl}, 2 \mathrm{NaCl}, 2 \mathrm{MgCl}_{2}, 10 \mathrm{Na}$-HEPES, 1 EGTA, $2 \mathrm{MgATP}$, and $0.3 \mathrm{Na}_{2} \mathrm{GTP}$ (pH 7.3) pH adjusted with $\mathrm{KOH}(290 \mathrm{mOsm})$. For recordings performed in voltage-clamp mode, the intracellular solution contained the following (in $\mathrm{mM}$ ): $125 \mathrm{CsMeSO}_{3}, 2 \mathrm{CsCl}, 10 \mathrm{Na}$ HEPES, 5 EGTA, $2 \mathrm{MgCl}_{2}$, and $4 \mathrm{MgATP}$. Signals were amplified using a Multiclamp 200B patch-clamp amplifier (Molecular Devices), filtered and digitized at $4 \mathrm{kHz}$ with an analogueto-digital converter (Digidata 1322A, Axon Instruments) and stored using pClamp 9 
software (Axon Instruments). The analysis of electrophysiological recordings was performed using Clampfit 10.2 (Molecular Devices) and MiniAnalysis software (Synaptsoft).

Passive membrane properties were obtained immediately after membrane rupture. Series resistances (range: $\sim 10-22 \mathrm{M} \Omega$, mean $\pm \mathrm{SD}: 16.3 \pm 2.6 \mathrm{M} \Omega$ ) were continuously monitored and recordings were discarded when these parameters changed by $>20 \%$. To record spontaneous and miniature inhibitory and excitatory post-synaptic currents (sIPSCs, mIPSCs, sEPSCs and mEPSCs, respectively), cells were held at $0 \mathrm{mV}$ and $-75 \mathrm{mV}$, respectively (correction of the liquid junction potential included). These values were chosen accordingly to the reversion potentials of glutamatergic and GABAergic currents. Miniature currents were recorded in the presence of TTX $(1 \mu \mathrm{M})$. Firing pattern were assessed in current clamp mode by injecting current in $25 \mathrm{pA}$ steps of $600 \mathrm{~ms}$ of duration starting from $-50 \mathrm{pA}$ to $225 \mathrm{pA}$. The firing frequency was determined at each step. For some experiments, PVIs were recorded before and $15 \mathrm{~min}$ after treatment with NRG1P $(10 \mathrm{nM})$ or CNO $(1 \mu \mathrm{M})$, or a mix of APV $(50 \mu \mathrm{M})$, DNQX $(10 \mu \mathrm{M})$ and Gabazine $(10 \mu \mathrm{M})$.

\section{Surgery}

Analgesic treatment (paracetamol $0.2 \mathrm{~g} / \mathrm{kg}$ ) was administered for two days prior to the surgery. Anaesthesia was induced at 5\% and maintained at 1.5-2\% isoflurane (w/v) (Baxter AG). Mice were placed in a stereotaxic apparatus (Angle One) above a heating pad. Eyes were covered with ointment (Lacyvisc, Alcon) and Lidocaine, a local analgesic, was injected below the skin overlaying the skull (s.c.). After exposing the skull, a craniotomy ( $3 \mathrm{~mm}$ in diameter using a biopsy punch from Miltex) was made above the hippocampus. In $P_{\text {valb }}{ }^{\text {cre/+}} ;$ Lgdel/+ mice, AAV infections $(0.1 \mu \mathrm{l}$ at $0.05 \mathrm{ml} / \mathrm{min})$ were performed using glass micropipettes (10-15 $\mu \mathrm{m}$ tip diameter; Drummond Scientific Company) at AP: $-1.7 \mathrm{~mm}$, ML: $1.0 \mathrm{~mm}$ bilaterally and DV: $1.5 \mathrm{~mm}$ relative to Bregma and the skull surface in order to target the dorsal CA1 area of the hippocampus. A few infections $(0.5 \mu \mathrm{l})$ were carried out using the coordinates AP: $-1.5 \mathrm{~mm}$, ML: $1.5 \mathrm{~mm}$ bilaterally and DV: $1.5 \mathrm{~mm}$ relative to Bregma and the skull surface. The micropipettes were left in place for $5 \mathrm{~min}$ after microinjection and slowly retracted $(0.4 \mathrm{~mm} / \mathrm{min})$ to avoid reflux of the viral solution. In all mice used for in-vivo electrophysiological recordings, the craniotomy was covered by a glass cover slip (Ø $3 \mathrm{~mm}$, Warner), which was then glued (Cyanocrylate, Cyberbond) to the skull. A ground wire was positioned a couple of millimetres posterior to the recording craniotomy at the surface of the cortex. A head-post, enabling head restraining during electrophysiological recordings ${ }^{62}$, was cemented to the skull using a mixture of dental cement (Jet Repair, Lang) and glue (Cyanocrylate, Cyberbond). AAV-Injected mice were used either for behavioural or electrophysiological measurements at least three weeks after infection. All mice used for electrophysiological recordings had at least three days for recovery and three days of head restraining habituation ${ }^{63}$. After the completion of each in vivo experiment, mice were injected i.p. with a lethal dose of pentobarbital $(150 \mathrm{mg} / \mathrm{kg})$ followed by intracardiac perfusion with $4 \%$ paraformaldehyde (PFA). Brains were maintained in 4\% PFA for one day and stored in PBS for histological verification of the polytrode position and/or infection accuracy (Supplementary Fig. 7). 


\section{In vivo electrophysiological recordings}

Animals were prepared with a subcutaneous catheter (if planned for s.c. injections) and then fixed to a head-restraining device as previously described ${ }^{64,65}$. The cover slip protecting the brain was then taken out and the dura-mater removed under $1.5 \%$ isoflurane anaesthesia. After stopping the anaesthesia flux, a DiI (Thermo Fisher Scientific) covered polytrode (Buszaki 64L; Neuronexustech, USA) was slowly lowered ( 15 min) between 1.2 and 1.7 $\mathrm{mm}$ DV from the surface of the cortex. Then, a period dedicated to the stabilization of the signal lasted between 10 to $20 \mathrm{~min}$. Broadband signals $(0.1-9000 \mathrm{~Hz})$ were acquired at 32 $\mathrm{KHz}$ sampling rate (Neuralynx Co) ${ }^{66}$. The first experiment consisted in recording a baseline period of $30 \mathrm{~min}$ followed by a s.c. injection of Neuregulin (NRG1P, $50 \mu \mathrm{g} / \mathrm{kg}$ ) and an additional hour period of drug induced neuronal activity. The second experiment consisted in injecting clozapine $\mathrm{N}$-oxide $(\mathrm{CNO}, 2 \mathrm{mg} / \mathrm{kg})$ i.p. before setting up the animals in the headrestraining device. The periods considered as control and as CNO-mediated excitation effect (magenta and green conditions in Fig. 6, respectively) consisted in 30-60 and 60-90 minutes post-injection, respectively. In both experiments, recordings were done for 1.5 hour. After completion of the experiments, the polytrode was slowly retracted and the animals were removed from the head-restraining holders.

\section{Analysis of the in vivo electrophysiological data}

Using the Matlab environment (MathWorks Co), local field potentials (LFP) were extracted by lowpass filtering $(1-90 \mathrm{~Hz})$ and downsampling $(200 \mathrm{~Hz})$ broadband signals. After a linear detrend of the signal, the LFP for each channel was normalized using z-scores. The spectral quantities were estimated with five tapers and a time-bandwidth product of three using the available toolbox named 'Chronux' (http://chronux.org). Time-frequency power spectra (window size of $10 \mathrm{~s}$ stepped each $2 \mathrm{~s}$ ) were calculated for the whole recordings. As the LFPs originating from the same shank were not independent, the median power of these LFPs was calculated to obtain a power estimate for each shank of the polytrode. Since various shanks may record different parts of CA1 with different levels of AAVs infections (Supplementary Fig. 7c), we analysed each shank independently and averaged the data across shank/mouse pairs. The time frequency power spectra were divided into epochs of 30 min. Each one of these epochs was represented by the median over time at each frequency bin.

Single unit activity (SUA) was obtained using the available python-based package named 'Klusta' and 'Klustaviewa' ${ }^{67}$. Shortly, the broadband signal was high-pass filtered at $500 \mathrm{~Hz}$ and negative peaks were extracted at 2.5 (weak) and 4.0 (strong) standard deviation (STD) thresholds. The waveforms around these peaks (referred to as "spikes") were extracted and clustered. Then, manual spike sorting enabled the isolation of SUAs (cluster quality $>0.95$ and similarities with other cluster $<0.05$ ) and only the LFPs recorded at shanks characterized by the presence of SUAs were kept for further analyses.

Then, neural synchrony was assessed using cross-correlations between the spike trains of two SUAs. Spike cross-correlation was calculated in a similar manner as the procedure used for the correlation of calcium onsets (see above). Spike trains were binned at $20 \mathrm{~ms}$ and the lags ranged from -600 to $600 \mathrm{~ms}$. Approximately $20 \%$ of neuronal pairs did not have 
coincident spiking within the chosen lag range and were excluded from the analyses. Epochs of $30 \mathrm{~min}$, as described above, were used to calculate the cross-correlations. The minimum number of pairs for each epoch was set at 10 and only recordings satisfying this minimum on all epochs were kept for further analyses (714 pairs per recording on average). The significance of the cross-correlation was evaluated by creating a surrogate population (500 iterations), which consisted in shifting one spike train by a random time between -600 and $600 \mathrm{~ms}$ (zero excluded). The threshold for significance was set at $0.00168,69$ with a false discovery rate correction for multiple testing of neuronal pairs and a Bonferroni correction for testing multiple time lags. In general, the cross-correlograms displayed a significant central peak around zero lag that varied in width. We chose to extract the maximum percentage of correlated pairs and to use these values as an index of synchrony. In one mouse (Lgdel/+), the percentage of correlated neurons was above 50\%, which is atypical and much higher than reported in the literature $\sim 15 \% 68,69$. This mouse was excluded from all the analyses.

\section{Behavioural testing}

All tests were performed in healthy adult mice (3-10 month old). After surgery was performed, rodents were left to rest for at least three weeks. Mice were habituated to a single experimenter by daily sessions of $5 \mathrm{~min}$ handling during the week prior to the behavioural test. To test for the influence of PV neurons on behavioural measures, vehicle $(\mathrm{NaCl} 0.9 \%)$, Neuregulin 1 peptide (NRG1P, $50 \mu \mathrm{g} / \mathrm{kg}$ ) or clozapine N-oxide $(\mathrm{CNO}, 2 \mathrm{mg} / \mathrm{kg}$ ) were administered i.p. as previously described ${ }^{70-72}$.

Open field test (OFT)—OFTs were performed under room lighting conditions (100 lux). Locomotor activity was recorded in a black Plexiglas box (43 $\mathrm{cm} \mathrm{L} \mathrm{x} 43 \mathrm{~cm} \mathrm{~W} \mathrm{x} 40 \mathrm{~cm} \mathrm{H}$ ) over which a Camera (Panasonic SD5) was placed. Mice were positioned into the centre of the arena and the distance travelled was measured during 10min using ANY-maze software (Stoelting, UK).

Contextual Fear Conditioning (cFC)—cFC was performed in a $15 \times 18 \mathrm{~cm}$ cage with a metallic grid floor. The walls were covered either with black/white stripes or black circle patterns to provide two different visual contexts. The conditioning sessions consisted in placing the mice during a total of $5 \mathrm{~min}$ in the cage. After $3 \mathrm{~min}$, three mild electric shocks $(0.8 \mathrm{~mA}, 2 \mathrm{~s})$ were applied through the floor grid with an interval of $30 \mathrm{~s}$. Then, mice were returned to their home cage. The next day, one testing session $(5 \mathrm{~min}$ ) was performed in the same cage but without electric shocks. Freezing behaviour was identified in video recordings using Anymaze software (Stoelting, UK) and manually verified by experimenters, who were blind to the experimental conditions.

\section{Measure of the acoustic startle reflex and the prepulse inhibition (PPI)-Mice}

were placed into a startle apparatus (Med-Associates, USA) consisting of a small metal and PVC cage inside a sound-attenuated chamber. Whole-body startle movements were quantified by an accelerometer mounted beneath the cage and stored by a computer using the Startle Reflex software (Med-Associates). Two loudspeakers were placed inside the sound-attenuated chamber: the first produced white noise acoustic stimuli, while the second 
produced a continuous $70 \mathrm{~dB}$ background noise throughout the test. The test began with 5 min acclimation to the apparatus, followed by presentation of different types of trials: pulse alone, prepulse alone, prepulse and pulse and no stimulus trials. Pulse- alone trials consisted of a $38 \mathrm{~ms}$ startle pulse at $120 \mathrm{~dB}$; prepulse-alone trials consisted of an $18 \mathrm{~ms}$ prepulse at 74 , 78,82 , or $86 \mathrm{~dB}$; prepulse-pulse trials consisted of a prepulse followed by the pulse. $100 \mathrm{~ms}$ separated the onsets between the two stimuli; and no-stimulus trials consisted of the background noise only. The test was divided in 3 blocks: block 1 consisted of 10 pulse-only trials, block 2 of 84 trials presented in pseudorandom order (12 pulse-only, 12 prepulsepulse of each intensity, 3 prepulse-only of each intensity, and 12 no-stimulus trials), and block 3 of 10 pulse-only trials.

Trials were presented in pseudorandom order, with a random intertrial interval of $10-15 \mathrm{~s}$. Startle response was determined as the absolute maximum-to-minimum value within $200 \mathrm{~ms}$ after the startle pulse onset. The entire test lasted $30 \mathrm{~min}$. The percentage PPI for each prepulse intensity level was calculated as follows: [100 - $100 \mathrm{x}$ (mean startle amplitude of prepulse-pulse trial/mean startle amplitude of pulse-alone trials)].

\section{Statistics}

Comparable sample size as in previously published studies was used. All statistical analyses were done with Matlab, Origin pro 8.0 or the Prism software. We used ANOVA and posthoc test, Kolmogorov-Smirnov test, Mann-Whitney test and paired Wilcoxon test. All tests were two-sided. Data distribution was assumed to be normal but this was not formally tested. For ANOVA, homogeneity of variance was tested using the Mauschly's sphericity test (subsequent Greenhouse-Geisser or Huynd-Feldt corrections were applied if sphericity criterion was not met). No statistical methods were used to pre-determine sample sizes but our sample sizes are similar to those reported in previous publications (see main text).

\section{Supplementary Material}

Refer to Web version on PubMed Central for supplementary material.

\section{Acknowledgements}

This paper is dedicated to the memory of D. Müller, whose ideas inspired this study and will continue to inspire all of us. We thank R. Kucherlapati (Harvard University) for generously providing the Lgdel/+ mice. We thank L. Jourdain and M.-P. Hervé for their technical support. We thank Y.Bernardelli, P. Mendez Garcia, T. Stefanelli, S. Eliez, P. Caroni and other members of the NCCR SYNAPSY for helpful discussions and/or comments on the manuscript. This research was supported by the University of Geneva, the Swiss National Science Foundation (grant numbers: 31003A_172878 to A.C., 310030B_144080 to D.M. and 310030E_135910 to I.R.), the National Center of Competence in Research (NCCR) "SYNAPSY - The Synaptic Bases of Mental Diseases" financed by the Swiss National Science Foundation (grant 51NF40-158776, D.M. and A.C.) and the Lejeune Foundation (T.M.).

\section{References}

1. Uhlhaas PJ, Singer W. Abnormal neural oscillations and synchrony in schizophrenia. Nat Rev Neurosci. 2010; 11:100-113. [PubMed: 20087360]

2. Stephan KE, Friston KJ, Frith CD. Dysconnection in schizophrenia: from abnormal synaptic plasticity to failures of self-monitoring. Schizophr Bull. 2009; 35:509-527. [PubMed: 19155345] 
3. Sigurdsson T, Stark KL, Karayiorgou M, Gogos JA, Gordon JA. Impaired hippocampal-prefrontal synchrony in a genetic mouse model of schizophrenia. Nature. 2010; 464:763-767. [PubMed: 20360742]

4. Cardin JA, et al. Driving fast-spiking cells induces gamma rhythm and controls sensory responses. Nature. 2009; 459:663-667. [PubMed: 19396156]

5. Amilhon B, et al. Parvalbumin Interneurons of Hippocampus Tune Population Activity at Theta Frequency. Neuron. 2015; 86:1277-1289. [PubMed: 26050044]

6. Gulyas AI, et al. Parvalbumin-containing fast-spiking basket cells generate the field potential oscillations induced by cholinergic receptor activation in the hippocampus. J Neurosci. 2010; 30:15134-15145. [PubMed: 21068319]

7. Cho KK, et al. Gamma rhythms link prefrontal interneuron dysfunction with cognitive inflexibility in Dlx5/6(+/-) mice. Neuron. 2015; 85:1332-1343. [PubMed: 25754826]

8. Gonzalez-Burgos G, Cho RY, Lewis DA. Alterations in cortical network oscillations and parvalbumin neurons in schizophrenia. Biol Psychiatry. 2015; 77:1031-1040. [PubMed: 25863358]

9. Lewis DA, Curley AA, Glausier JR, Volk DW. Cortical parvalbumin interneurons and cognitive dysfunction in schizophrenia. Trends Neurosci. 2012; 35:57-67. [PubMed: 22154068]

10. Steullet $\mathrm{P}$, et al. Oxidative stress-driven parvalbumin interneuron impairment as a common mechanism in models of schizophrenia. Molecular psychiatry. 2017

11. Sauer JF, Struber M, Bartos M. Impaired fast-spiking interneuron function in a genetic mouse model of depression. eLife. 2015; 4

12. Nguyen R, et al. Parvalbumin and GAD65 interneuron inhibition in the ventral hippocampus induces distinct behavioral deficits relevant to schizophrenia. J Neurosci. 2014; 34:14948-14960. [PubMed: 25378161]

13. Del Pino I, et al. Erbb4 deletion from fast-spiking interneurons causes schizophrenia-like phenotypes. Neuron. 2013; 79:1152-1168. [PubMed: 24050403]

14. Belforte JE, et al. Postnatal NMDA receptor ablation in corticolimbic interneurons confers schizophrenia-like phenotypes. Nature neuroscience. 2010; 13:76-83. [PubMed: 19915563]

15. Korotkova T, Fuchs EC, Ponomarenko A, von Engelhardt J, Monyer H. NMDA receptor ablation on parvalbumin-positive interneurons impairs hippocampal synchrony, spatial representations, and working memory. Neuron. 2010; 68:557-569. [PubMed: 21040854]

16. Carlen M, et al. A critical role for NMDA receptors in parvalbumin interneurons for gamma rhythm induction and behavior. Molecular psychiatry. 2012; 17:537-548. [PubMed: 21468034]

17. Bhugra D. The global prevalence of schizophrenia. PLoS Med. 2005; 2:e151. [PubMed: 15916460]

18. Karayiorgou M, Simon TJ, Gogos JA. 22q11.2 microdeletions: linking DNA structural variation to brain dysfunction and schizophrenia. Nat Rev Neurosci. 2010; 11:402-416. [PubMed: 20485365]

19. Karayiorgou M, et al. Schizophrenia susceptibility associated with interstitial deletions of chromosome 22q11. Proceedings of the National Academy of Sciences of the United States of America. 1995; 92:7612-7616. [PubMed: 7644464]

20. Moutin E, et al. Palmitoylation of cdc42 Promotes Spine Stabilization and Rescues Spine Density Deficit in a Mouse Model of 22q11.2 Deletion Syndrome. Cereb Cortex. 2016

21. Stark KL, et al. Altered brain microRNA biogenesis contributes to phenotypic deficits in a 22q11deletion mouse model. Nat Genet. 2008; 40:751-760. [PubMed: 18469815]

22. Piskorowski RA, et al. Age-Dependent Specific Changes in Area CA2 of the Hippocampus and Social Memory Deficit in a Mouse Model of the 22q11.2 Deletion Syndrome. Neuron. 2016; 89:163-176. [PubMed: 26748091]

23. Drew LJ, et al. Evidence for altered hippocampal function in a mouse model of the human 22q11.2 microdeletion. Mol Cell Neurosci. 2011; 47:293-305. [PubMed: 21635953]

24. Li KX, et al. Neuregulin 1 regulates excitability of fast-spiking neurons through Kv1.1 and acts in epilepsy. Nature neuroscience. 2011; 15:267-273. [PubMed: 22158511]

25. Harris KD, Csicsvari J, Hirase H, Dragoi G, Buzsaki G. Organization of cell assemblies in the hippocampus. Nature. 2003; 424:552-556. [PubMed: 12891358]

26. Jones CA, Watson DJ, Fone KC. Animal models of schizophrenia. Br J Pharmacol. 2011; 164:1162-1194. [PubMed: 21449915] 
27. Mena A, et al. Reduced Prepulse Inhibition as a Biomarker of Schizophrenia. Front Behav Neurosci. 2016; 10:202. [PubMed: 27803654]

28. Long JM, et al. Behavior of mice with mutations in the conserved region deleted in velocardiofacial/DiGeorge syndrome. Neurogenetics. 2006; 7:247-257. [PubMed: 16900388]

29. Swerdlow NR, Braff DL, Geyer MA. Sensorimotor gating of the startle reflex: what we said 25 years ago, what has happened since then, and what comes next. J Psychopharmacol. 2016; 30:1072-1081. [PubMed: 27539931]

30. Mier D, et al. Evidence for altered amygdala activation in schizophrenia in an adaptive emotion recognition task. Psychiatry Res. 2014; 221:195-203. [PubMed: 24434194]

31. Paylor R, et al. Mice deleted for the DiGeorge/velocardiofacial syndrome region show abnormal sensorimotor gating and learning and memory impairments. Hum Mol Genet. 2001; 10:26452650. [PubMed: 11726551]

32. Cannon TD. How Schizophrenia Develops: Cognitive and Brain Mechanisms Underlying Onset of Psychosis. Trends Cogn Sci. 2015; 19:744-756. [PubMed: 26493362]

33. Lieberman JA, et al. Hippocampal dysfunction in the pathophysiology of schizophrenia: a selective review and hypothesis for early detection and intervention. Molecular psychiatry. 2018

34. Zaremba JD, et al. Impaired hippocampal place cell dynamics in a mouse model of the 22q11.2 deletion. Nature neuroscience. 2017; 20:1612-1623. [PubMed: 28869582]

35. Stefansson H, et al. Neuregulin 1 and susceptibility to schizophrenia. Am J Hum Genet. 2002; 71:877-892. [PubMed: 12145742]

36. Mukai J, et al. Molecular substrates of altered axonal growth and brain connectivity in a mouse model of schizophrenia. Neuron. 2015; 86:680-695. [PubMed: 25913858]

37. Tamura M, Mukai J, Gordon JA, Gogos JA. Developmental Inhibition of Gsk3 Rescues Behavioral and Neurophysiological Deficits in a Mouse Model of Schizophrenia Predisposition. Neuron. 2016; 89:1100-1109. [PubMed: 26898776]

38. Hu H, Gan J, Jonas P. Interneurons. Fast-spiking, parvalbumin(+) GABAergic interneurons: from cellular design to microcircuit function. Science (New York, N.Y. 2014; 345

39. Yamada J, Jinno S. Molecular heterogeneity of aggrecan-based perineuronal nets around five subclasses of parvalbumin-expressing neurons in the mouse hippocampus. J Comp Neurol. 2017; 525:1234-1249. [PubMed: 27718219]

40. Klausberger T, et al. Spike timing of dendrite-targeting bistratified cells during hippocampal network oscillations in vivo. Nature neuroscience. 2004; 7:41-47. [PubMed: 14634650]

41. Lee SH, et al. Parvalbumin-positive basket cells differentiate among hippocampal pyramidal cells. Neuron. 2014; 82:1129-1144. [PubMed: 24836505]

42. Donato F, Chowdhury A, Lahr M, Caroni P. Early- and late-born parvalbumin basket cell subpopulations exhibiting distinct regulation and roles in learning. Neuron. 2015; 85:770-786. [PubMed: 25695271]

43. Viney TJ, et al. Network state-dependent inhibition of identified hippocampal CA3 axo-axonic cells in vivo. Nature neuroscience. 2013; 16:1802-1811. [PubMed: 24141313]

44. Howard A, Tamas G, Soltesz I. Lighting the chandelier: new vistas for axo-axonic cells. Trends Neurosci. 2005; 28:310-316. [PubMed: 15927687]

45. Curley AA, Lewis DA. Cortical basket cell dysfunction in schizophrenia. The Journal of physiology. 2012; 590:715-724. [PubMed: 22219337]

46. Bartos M, et al. Fast synaptic inhibition promotes synchronized gamma oscillations in hippocampal interneuron networks. Proceedings of the National Academy of Sciences of the United States of America. 2002; 99:13222-13227. [PubMed: 12235359]

47. Katona I, Acsady L, Freund TF. Postsynaptic targets of somatostatin-immunoreactive interneurons in the rat hippocampus. Neuroscience. 1999; 88:37-55. [PubMed: 10051188]

48. Acsady L, Gorcs TJ, Freund TF. Different populations of vasoactive intestinal polypeptideimmunoreactive interneurons are specialized to control pyramidal cells or interneurons in the hippocampus. Neuroscience. 1996; 73:317-334. [PubMed: 8783252]

49. Harris KD, et al. Classes and continua of hippocampal CA1 inhibitory neurons revealed by singlecell transcriptomics. bioRxiv. 2018 
50. Hamm JP, Peterka DS, Gogos JA, Yuste R. Altered Cortical Ensembles in Mouse Models of Schizophrenia. Neuron. 2017; 94:153-167 e158. [PubMed: 28384469]

51. Merscher S, et al. TBX1 is responsible for cardiovascular defects in velo-cardio-facial/DiGeorge syndrome. Cell. 2001; 104:619-629. [PubMed: 11239417]

52. Hippenmeyer $\mathrm{S}$, et al. A developmental switch in the response of DRG neurons to ETS transcription factor signaling. PLoS Biol. 2005; 3:e159. [PubMed: 15836427]

53. Kastin AJ, Akerstrom V, Pan W. Neuregulin-1-beta1 enters brain and spinal cord by receptormediated transport. Journal of neurochemistry. 2004; 88:965-970. [PubMed: 14756818]

54. Rosler TW, et al. Biodistribution and brain permeability of the extracellular domain of neuregulin-1-beta1. Neuropharmacology. 2011; 61:1413-1418. [PubMed: 21903113]

55. Armbruster BN, Li X, Pausch MH, Herlitze S, Roth BL. Evolving the lock to fit the key to create a family of G protein-coupled receptors potently activated by an inert ligand. Proceedings of the National Academy of Sciences of the United States of America. 2007; 104:5163-5168. [PubMed: 17360345]

56. Stoppini L, Buchs PA, Muller D. A simple method for organotypic cultures of nervous tissue. Journal of neuroscience methods. 1991; 37:173-182. [PubMed: 1715499]

57. Chen TW, et al. Ultrasensitive fluorescent proteins for imaging neuronal activity. Nature. 2013; 499:295-300. [PubMed: 23868258]

58. Urban DJ, Roth BL. DREADDs (designer receptors exclusively activated by designer drugs): chemogenetic tools with therapeutic utility. Annual review of pharmacology and toxicology. 2015; 55:399-417.

59. Fisahn A, Pike FG, Buhl EH, Paulsen O. Cholinergic induction of network oscillations at $40 \mathrm{~Hz}$ in the hippocampus in vitro. Nature. 1998; 394:186-189. [PubMed: 9671302]

60. Pietersen AN, et al. Transition between fast and slow gamma modes in rat hippocampus area CA1 in vitro is modulated by slow CA3 gamma oscillations. The Journal of physiology. 2014; 592:605620. [PubMed: 24277864]

61. Marissal T, et al. Pioneer glutamatergic cells develop into a morpho-functionally distinct population in the juvenile CA3 hippocampus. Nature communications. 2012; 3

62. Gschwend O, et al. Neuronal pattern separation in the olfactory bulb improves odor discrimination learning. Nature neuroscience. 2015; 18:1474-1482. [PubMed: 26301325]

63. Abraham NM, Guerin D, Bhaukaurally K, Carleton A. Similar odor discrimination behavior in head-restrained and freely moving mice. PLoS One. 2012; 7:e51789. [PubMed: 23272168]

64. Gschwend O, Beroud J, Carleton A. Encoding odorant identity by spiking packets of rate-invariant neurons in awake mice. PLoS ONE. 2012; 7:e30155. [PubMed: 22272291]

65. Gschwend O, Beroud J, Vincis R, Rodriguez I, Carleton A. Dense encoding of natural odorants by ensembles of sparsely activated neurons in the olfactory bulb. Scientific reports. 2016; 6

66. Patterson MA, Lagier S, Carleton A. Odor representations in the olfactory bulb evolve after the first breath and persist as an odor afterimage. Proceedings of the National Academy of Sciences of the United States of America. 2013; 110:E3340-3349. [PubMed: 23918364]

67. Rossant C, et al. Spike sorting for large, dense electrode arrays. Nature neuroscience. 2016; 19:634-641. [PubMed: 26974951]

68. Csicsvari J, Hirase H, Czurko A, Buzsaki G. Reliability and state dependence of pyramidal cellinterneuron synapses in the hippocampus: an ensemble approach in the behaving rat. Neuron. 1998; 21:179-189. [PubMed: 9697862]

69. Bartho $\mathrm{P}$, et al. Characterization of neocortical principal cells and interneurons by network interactions and extracellular features. Journal of neurophysiology. 2004; 92:600-608. [PubMed: 15056678]

70. Engel M, et al. Neuregulin 1 Prevents Phencyclidine-Induced Behavioral Impairments and Disruptions to GABAergic Signaling in Mice. Int J Neuropsychopharmacol. 2015; 18

71. Stefanelli T, Bertollini C, Luscher C, Muller D, Mendez P. Hippocampal Somatostatin Interneurons Control the Size of Neuronal Memory Ensembles. Neuron. 2016; 89:1074-1085. [PubMed: 26875623] 
72. Alexander GM, et al. Remote control of neuronal activity in transgenic mice expressing evolved G protein-coupled receptors. Neuron. 2009; 63:27-39. [PubMed: 19607790] 

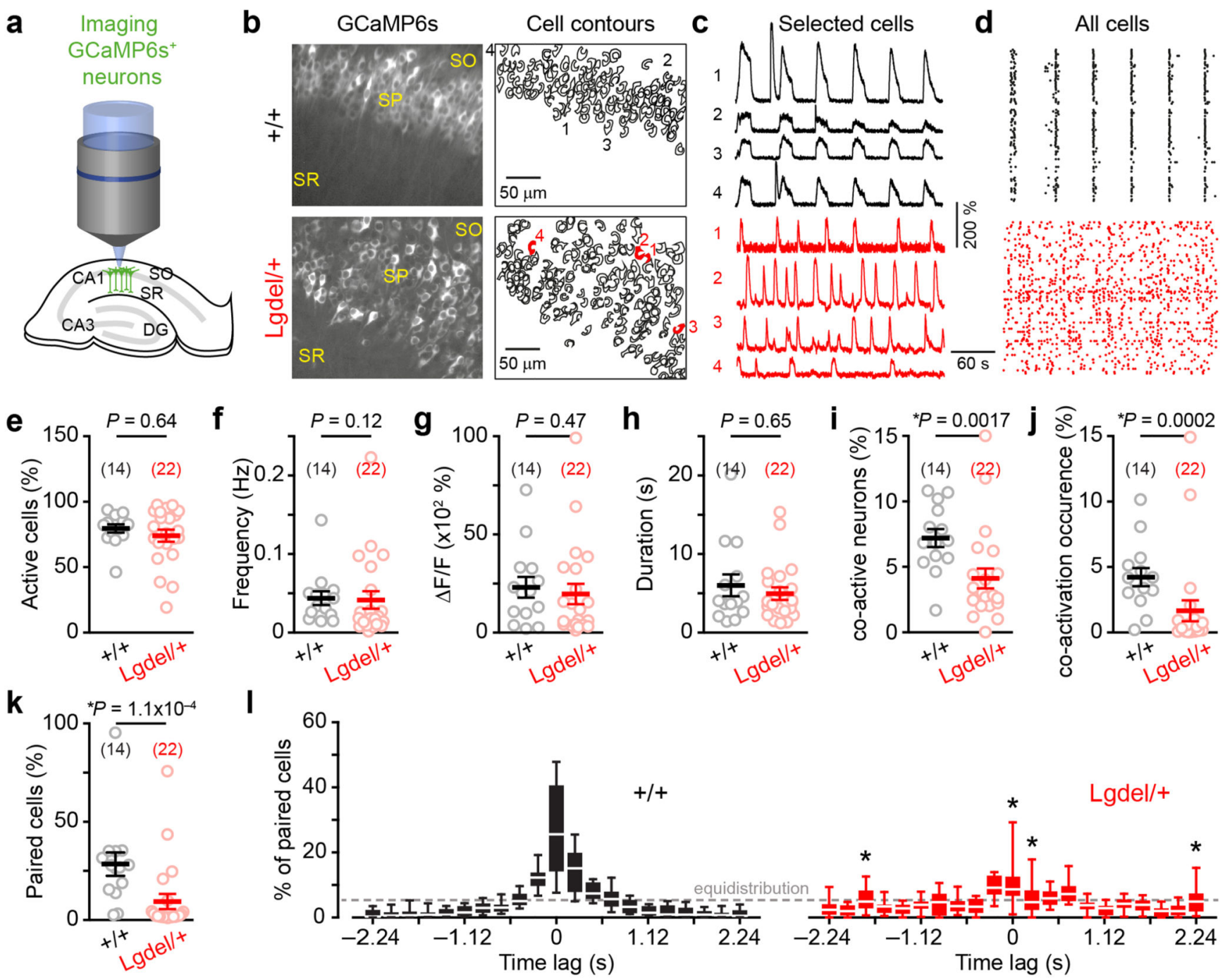

Fig. 1. Desynchronization of CA1 neural assemblies in Lgdel/+ mice.

(a) Schema of the experimental procedure. CA: cornu ammonis, DG: dentate gyrus, SO: stratum oriens, SP: stratum pyramidale, SR: stratum radiatum. (b) Photographs and contours of GCaMP6s-expressing neurons in the CA1 region of WT and Lgdel/+ cultured slices (experiment was repeated independently with similar results, see e-h for quantification). (c) Examples of $\mathrm{Ca}^{2+}$ sweeps recorded in presence of carbachol $(50 \mu \mathrm{M})$ from selected neurons identified as 1 to 4 in $\mathbf{b}$. (d) Raster plots of $\mathrm{Ca}^{2+}$ transient onsets extracted from all neurons shown in b. (e) Proportion of neurons displaying $\mathrm{Ca}^{2+}$ transients in the $\mathrm{CA} 1$ region (each circle represents the mean for a given slice, number of slices indicated in parentheses). (f-h) Frequency (f), amplitude (g) and duration (h) of $\mathrm{Ca}^{2+}$ transients recorded in neurons of the CA1 area. The mean frequency did not differ significantly despite a trend for a more prominent subpopulation of neurons with low firing rate in Lgdel/+ mice compared to WT mice (Supplementary Fig. 1a,b). (i-j) Percentage of co-active neurons above random coactivation (I) and occurrence of these co-activations (j). (k) Percentage of correlated pairs of neurons above chance. (l) Distributions of significant time lags in correlated pairs. Box: 
25-75 th percentiles, whiskers: $10-90^{\text {th }}$ percentiles, white bar: median. Statistical comparisons were done either with a two-sided Mann-Whitney test (e-k) or a two-way repeated measures ANOVA (l, interaction genotype x time lags $F_{20,680}=9.95, P<10^{-15}$ ) followed by Bonferroni post-hoc test $\left(\mathbf{l},{ }^{*} P<0.05\right.$ : see Supplementary Table 1 for detailed statistics). Data are presented as mean \pm SEM unless indicated otherwise. 

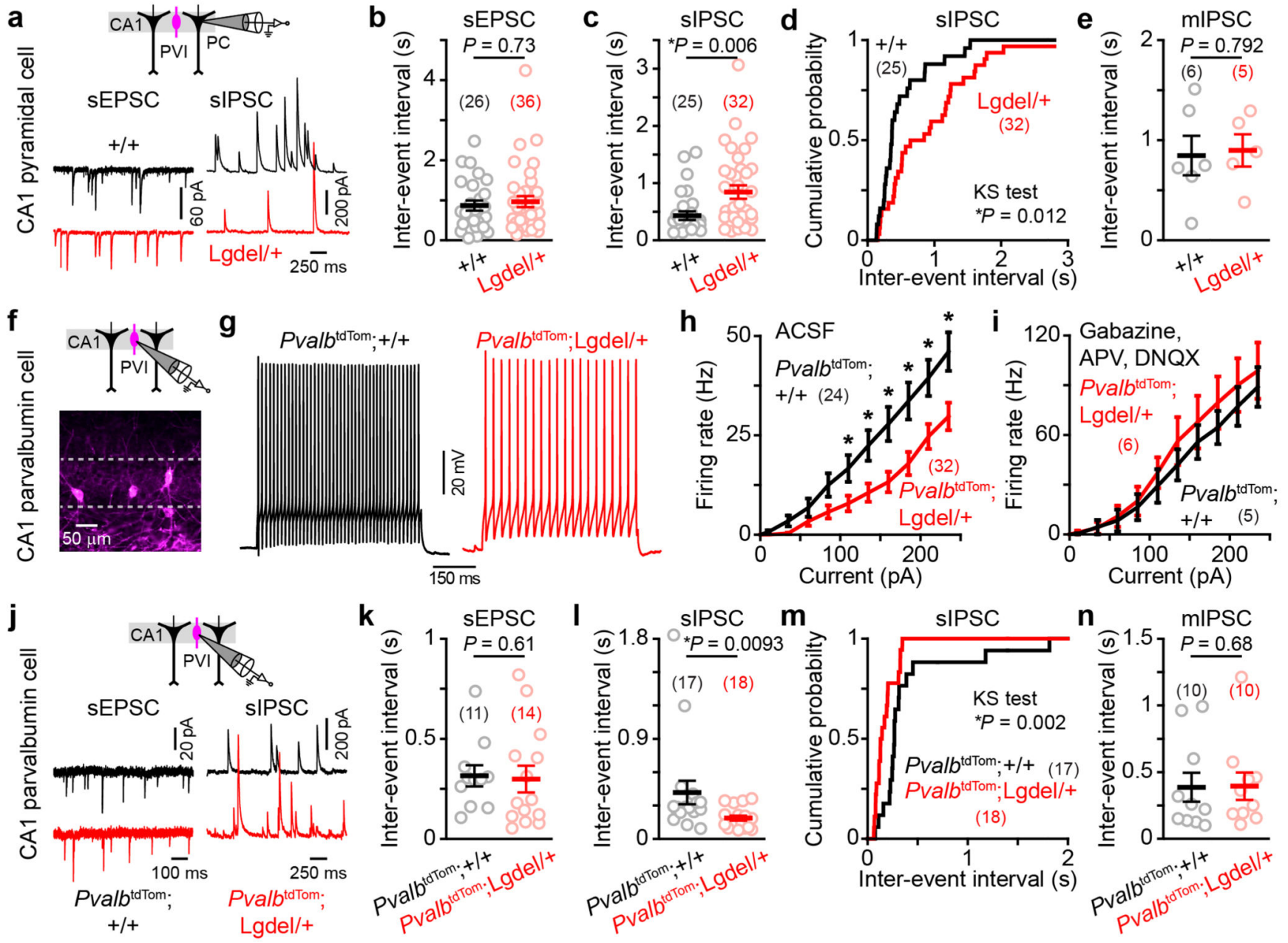

Fig. 2. Reduced inhibitory inputs to CA1 pyramidal cells and hypoexcitability of CA1 PVIs in Lgdel/+ mice.

(a-e) Patch-clamp recordings of CA1 pyramidal cells (PC). (a) Examples of voltage-clamp recordings showing either spontaneous excitatory (sEPSC) or spontaneous inhibitory (sIPSC) post-synaptic currents recorded from PC. (b) Mean sEPSC inter-event interval (IEI). (c-d) Summary graph (c) and cumulative distribution (d) of the mean sIPSC IEI (KS:

Kolmogorov-Smirnov test). (e) Mean miniature IPSC IEI. (f-n) Patch-clamp recordings of CA1 PVIs from $P$ val $b^{\text {cre/+}} ;+/+$ and $P$ val $b^{\text {cre/++}} ;$ Lgdel/+ slices injected with AAV allowing the conditional expression of tdTomato $\left(P\right.$ val $\left.b^{\text {tdTom }}, \mathbf{f}\right)$. (g) Representative spiking activity evoked by the same depolarizing step of current in CA1 PVIs. (h-i) Input/output functions for CA1 PVIs in either artificial cerebral spinal fluid (ACSF, $\mathbf{h}$; two-way repeated measures ANOVA: genotype effect $F_{1,54}=9.7, P=0.003$; two-sided Fisher post-hoc test $* P<0.05$ : see Supplementary Table 1 for detailed statistics) or in blockers of the fast glutamatergic and GABAergic synaptic transmission (i, two-way repeated measures ANOVA: genotype effect $\left.F_{1,9}=0.42, P=0.54\right)$. (j) Examples of sEPSC or sIPSC recorded from PVIs. (k) Mean sEPSC IEI. (l-m) Summary graph (l) and cumulative distribution (m) of the mean sIPSC IEI. (n) Mean mIPSC IEI. All data are presented as mean \pm SEM (each circle represents a 
cell, number of cells indicated in parentheses). Two-sided Mann-Whitney test used in b,c,e,k,l,n. 

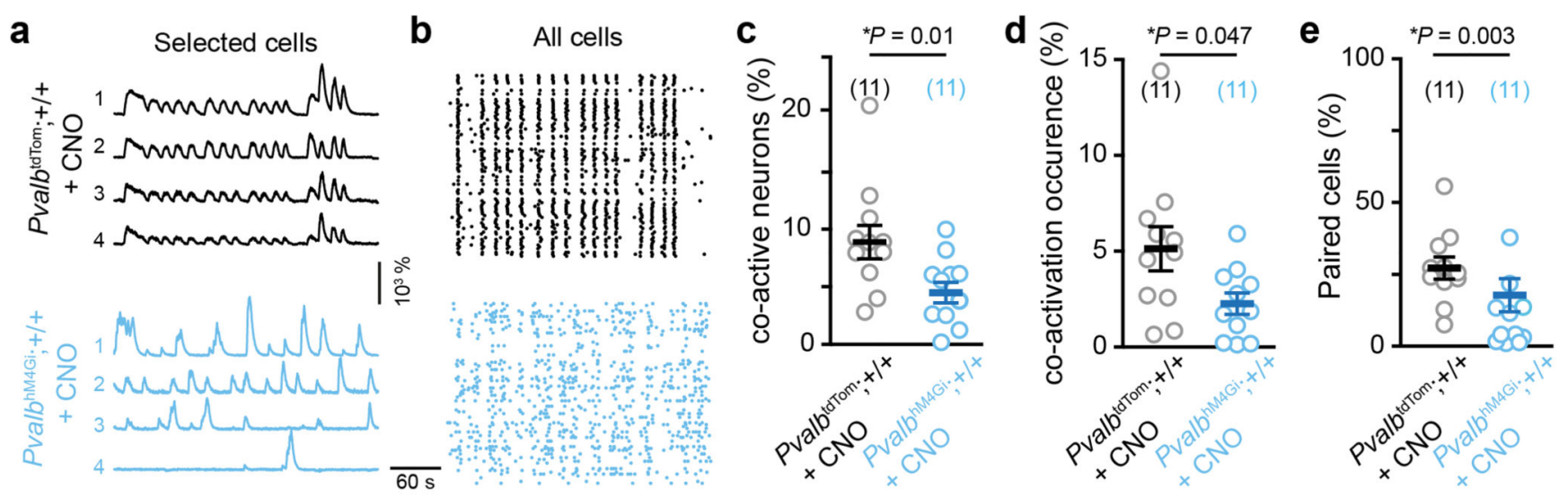

Fig. 3. Chemogenetic inhibition of PVI desynchronizes CA1 assemblies in WT mice.

(a) Examples of $\mathrm{Ca}^{2+}$ sweeps recorded after clozapine-N-oxide (CNO, $\left.1 \mu \mathrm{M}\right)$ bath application from four selected neurons in slices of $P_{\text {val }} b^{\text {cre/+}} ;+/+$ mice infected with AAVs enabling the conditional expression of either tdTomato $\left(P_{V a l} b^{\text {tdTom }} ;+/+\right)$ or the inhibitory DREADD hM4Gi ( $P$ vall $\left.b^{\mathrm{hM} 4 \mathrm{Gi}} ;+++\right)$. (b) Raster plots of $\mathrm{Ca}^{2+}$ transient onsets extracted from all neurons of an imaged field. (c-d) Percentage of co-active neurons above random coactivation (c) and occurrence of these co-activations (d). (e) Percentage of correlated pairs of neurons above chance. All data are presented as mean \pm SEM and compared with the twosided Mann-Whitney test. 

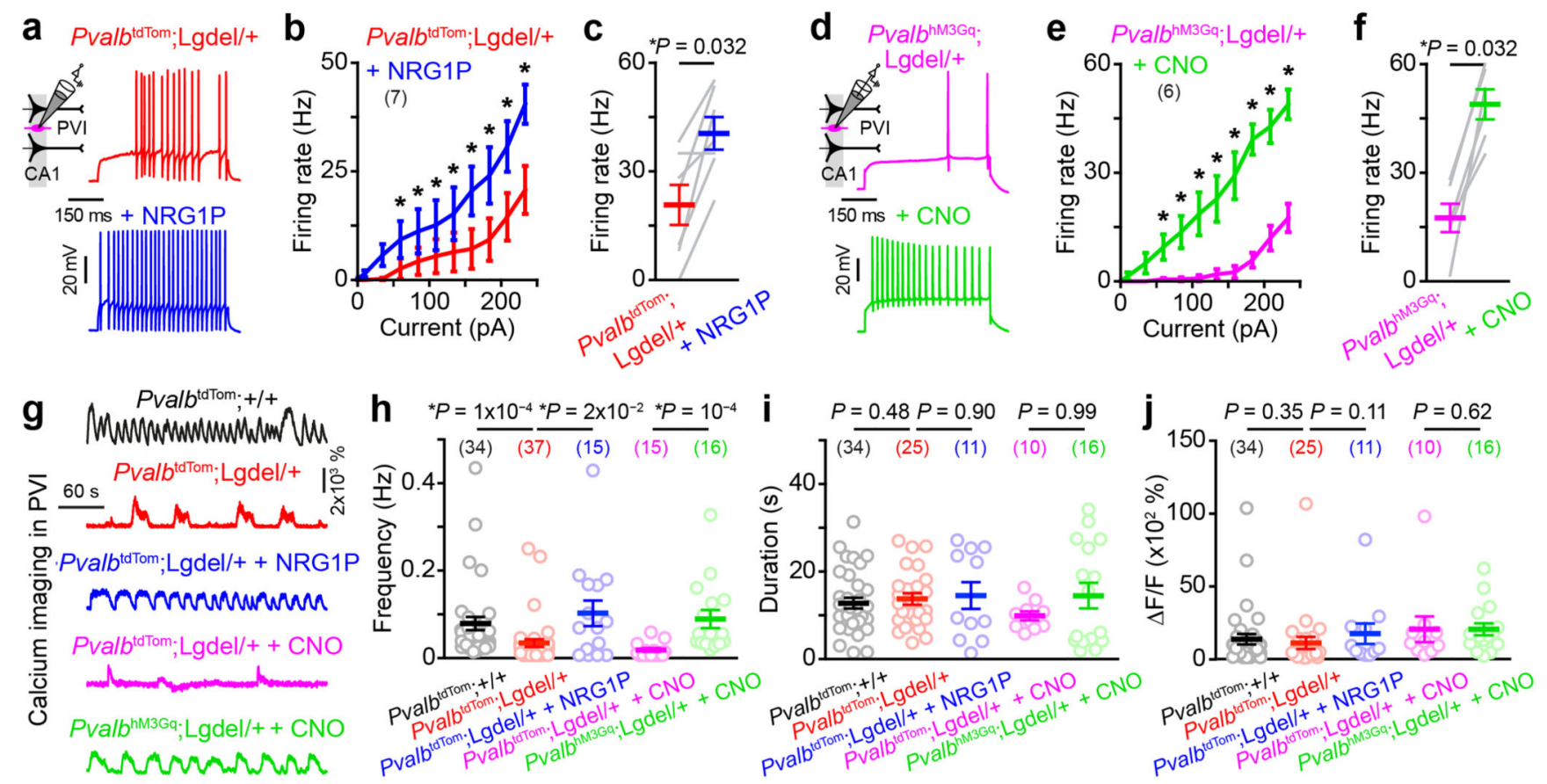

Fig. 4. Rescue of Lgdel/+ PVI excitability using pharmacological and chemogenetic strategies. (a) Examples of spiking activity evoked by the same depolarizing step of current in CA1 PVIs recorded from $P$ valb ${ }^{\text {cre/+}} ;$ Lgdel//+ mice before and after neuregulin 1 peptide bath application (NRG1P, $10 \mathrm{nM}$ ). (b) Input/output functions for CA1 PVIs recorded before and after NRG1P application (two-way repeated measures ANOVA: interaction treatment $\mathrm{x}$ injected current $F_{9,54}=3.54 P=0.0016$; Two-sided Fisher post-hoc test $* P<0.05$ : see Supplementary Table 1 for detailed statistics). (c) Summary graph of the firing frequency generated by PVIs in response to a 235 pA current step before and after NRG1P application (Two-sided Wilcoxon paired test). (d) Examples of spiking activity evoked by the same depolarizing step of current in CA1 PVIs recorded from $P$ val $b^{\text {cre/++}} ;$ Lgdel/+ expressing the excitatory DREADD hM3Gq ( $P$ val $\left.b^{\mathrm{hM} 3 \mathrm{Gq}} ; \mathrm{Lgdel} /+\right)$ before and after clozapine-N-oxide bath application $(\mathrm{CNO}, 1 \mu \mathrm{M})$. (e) Input/output functions for CA1 PVIs recorded before and after CNO application (two-way repeated measures ANOVA: interaction treatment $\mathrm{x}$ injected current $F_{9,45}=8.2 P=4.6 \times 10^{-7}$; Two-sided Fisher post-hoc test $* P<0.05$ : see Supplementary Table 1 for detailed statistics). (f) Summary graph of the firing frequency generated by PVIs in response to a $235 \mathrm{pA}$ current step before and after $\mathrm{CNO}$ application (Two-sided Wilcoxon paired test). (g) Examples of $\mathrm{Ca}^{2+}$ sweeps recorded from PVIs in various conditions. (h-j) Frequency (h), duration (i) and amplitude (j) of $\mathrm{Ca}^{2+}$ transients extracted from all imaged PVIs (numbers of imaged cells indicated in parentheses). Data are presented as mean \pm SEM and comparisons done with Two-sided Mann-Whitney test unless indicated otherwise. 


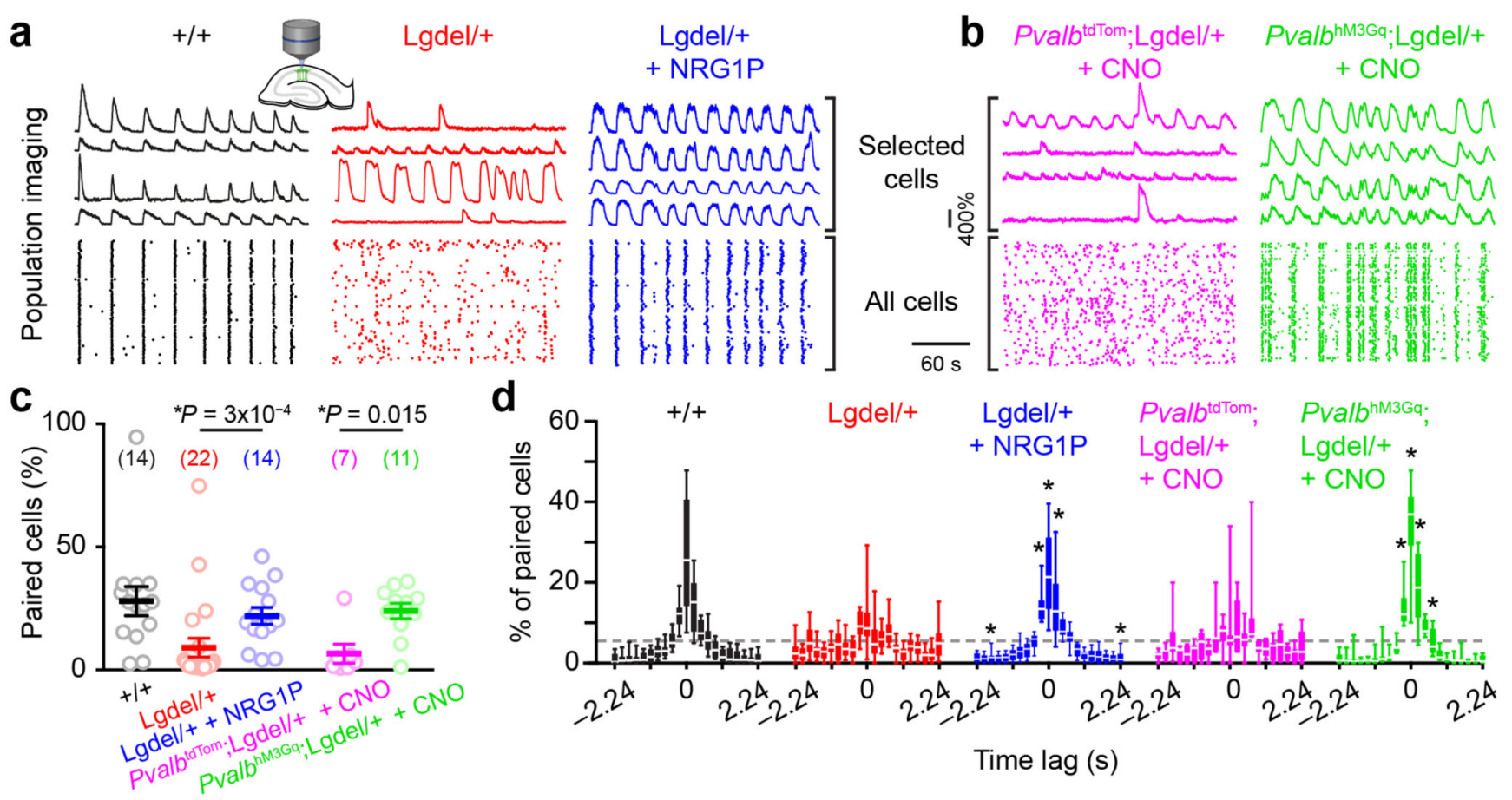

Fig. 5. Increasing PVI excitability restores synchronization of $\mathrm{CA1}$ assemblies in $\mathrm{Lgdel} / \mathrm{+}$ slices. (a-b) Examples of $\mathrm{Ca}^{2+}$ sweeps recorded from selected neurons and of raster plots of $\mathrm{Ca}^{2+}$ transient onsets extracted from all imaged neurons in slices from various genotypes and following various drug applications. (c) Percentage of correlated pairs of neurons (each circle represents a slice, number of slices indicated in parentheses; Two-Sided MannWhitney test; data are presented as mean \pm SEM). (d) Distributions of the time lag computed for correlated pairs (two-way repeated measures ANOVA with Bonferroni post-hoc test $* P<$ 0.05; ANOVA interaction genotype x time lags: $F_{20,660}=7.53 P<10^{-15}$ and $F_{20,320}=10.65$ $P<10^{-15}$ for NRG1P and CNO treatments, respectively; see Supplementary Table 1 for detailed statistics). Grey dashed line represents the equidistribution. Box: $25-75^{\text {th }}$ percentiles, whiskers: $10-90^{\text {th }}$ percentiles, white bar: median. 
a

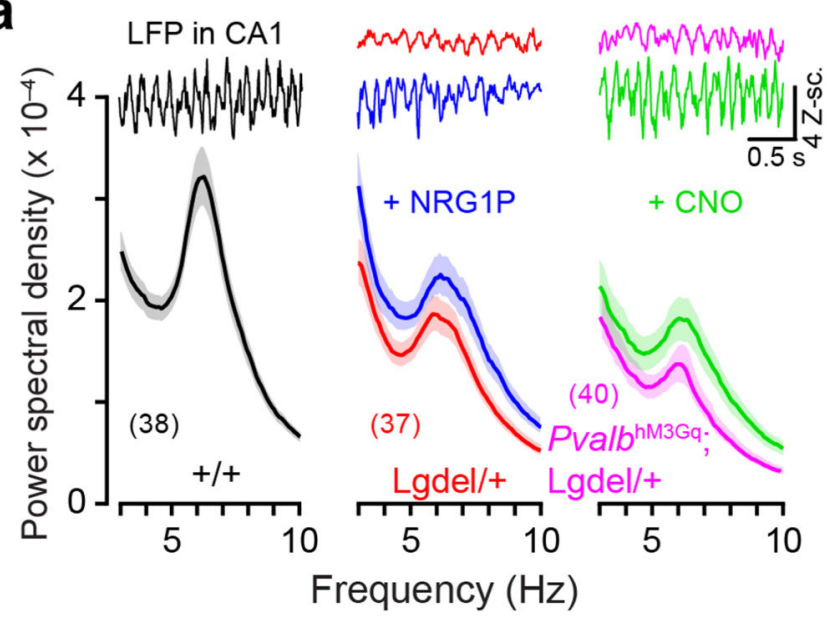

d

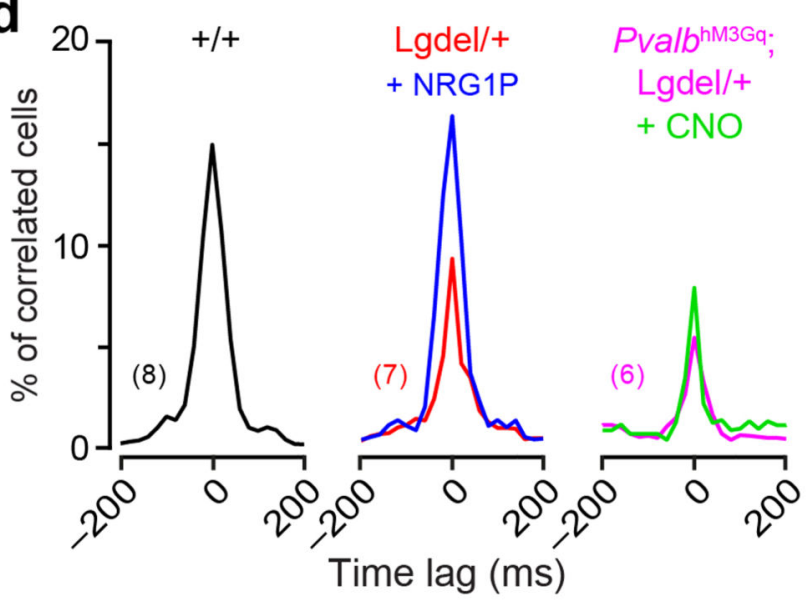

b
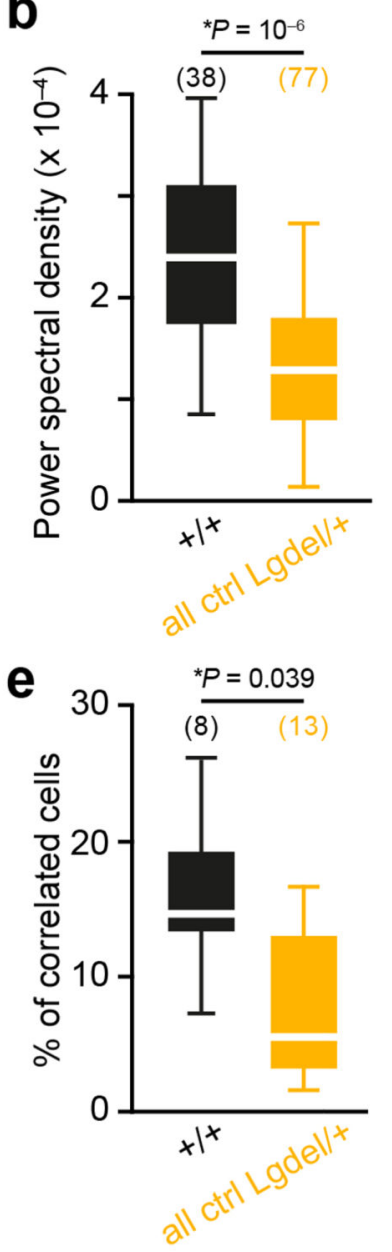

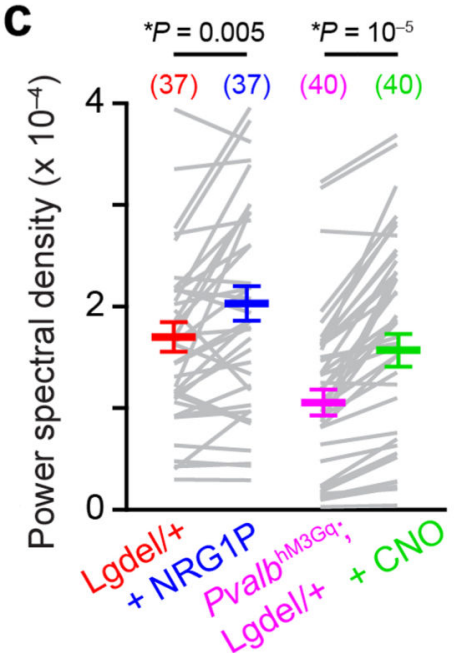

f

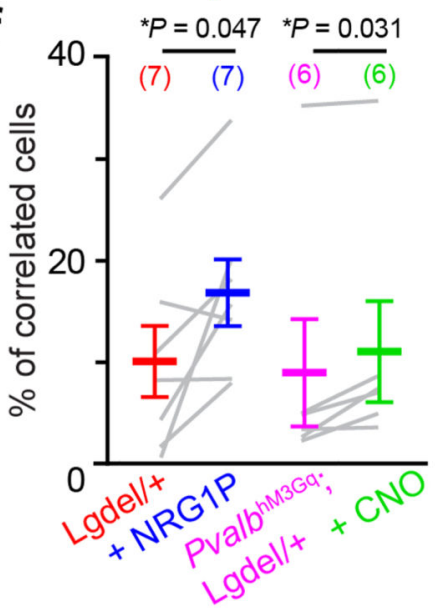

Fig. 6. Increasing PVI excitability during adulthood improves hippocampal-theta oscillations and neuronal correlations in vivo.

(a) Representative sweeps of local field potentials (LFPs) recorded in the CA1 region of awake mice. The distributions of LFP power spectral densities (PSDs) are presented between 3 and $10 \mathrm{~Hz}$ for visibility (see Supplementary Fig. 7 for the full frequency range; numbers in parentheses indicate the number of shank/mouse pairs). Data are plotted for mice of the following genotypes: WT, Lgdel/+ before and after NRG1P injection, Pvalb ${ }^{\mathrm{hM} 3 \mathrm{Gq}} ; \mathrm{Lgdel} /+$ before and after CNO induced effect. (b) Distribution of LFP PSDs for different groups (averaged between 5 to $8 \mathrm{~Hz}$ for the theta frequency band; two-sided Mann-Whitney test). Data from Lgdel/+ and $P$ Valb ${ }^{\mathrm{cre} /+} ; \mathrm{Lgdel} /+$ during the baseline were grouped and referred as Lgdel/+ control mice (all ctrl Lgdel/+). (c) PSDs in the theta frequency range in Lgdel/+ mice in the control conditions or after treatments with NRG1P (left) or CNO (right), respectively (two-sided Wilcoxon paired test). (d) Percentage of correlated neurons (median) in the CA1 region of awake mice as a function of time lags. The numbers of recorded animals are indicated in parentheses. (e) Distribution of central peaks of correlation curves (computed in $\pm 60 \mathrm{~ms}$ time window; two-sided Mann-Whitney test). (f) Effects of the different treatments on neuronal correlations (two-sided Wilcoxon paired test). Data 
presented as mean \pm SEM in a,c,f and as box plots in b,e (box: $25-75^{\text {th }}$ percentiles, whiskers: $10-90^{\text {th }}$ percentiles, white bar: median). 

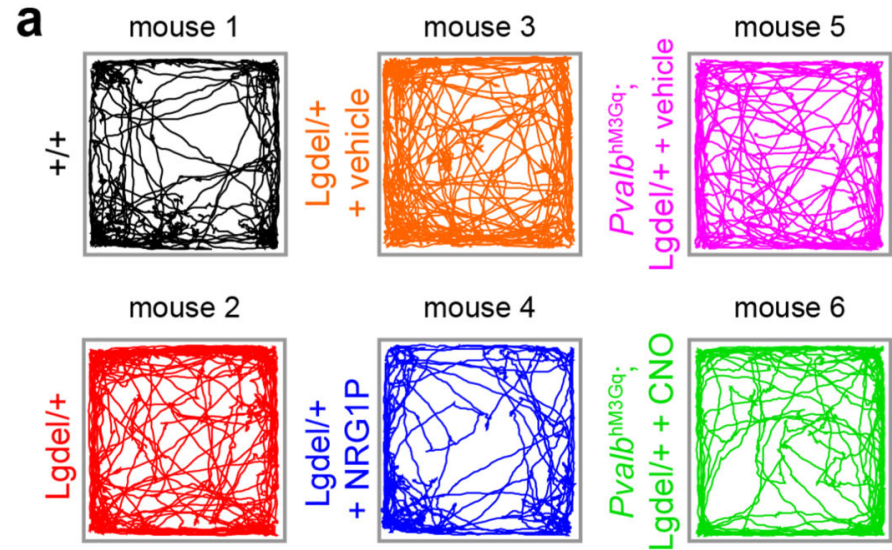

Open field test b

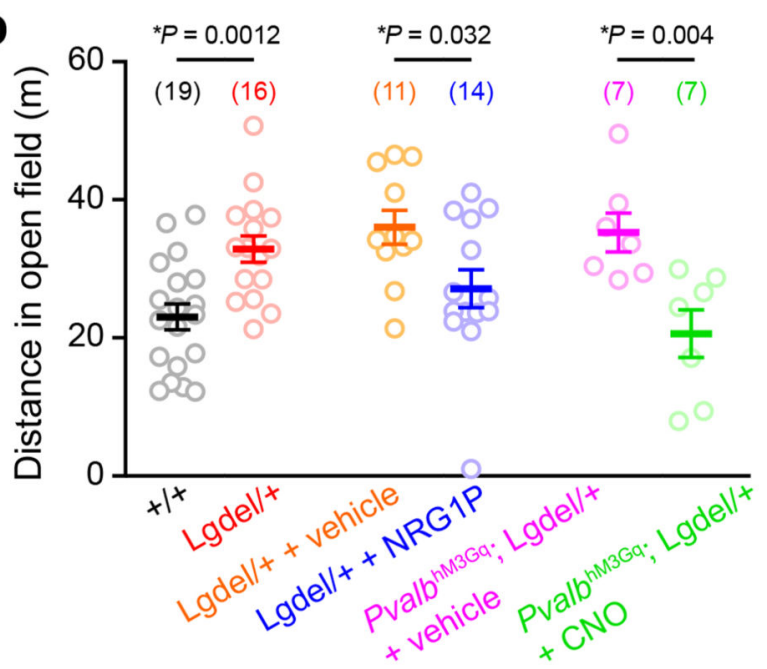

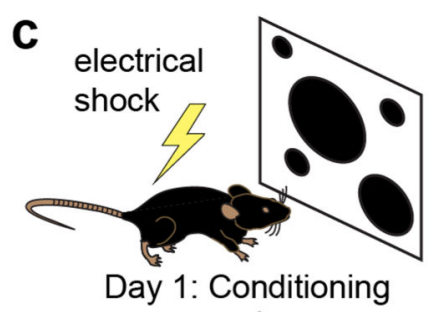

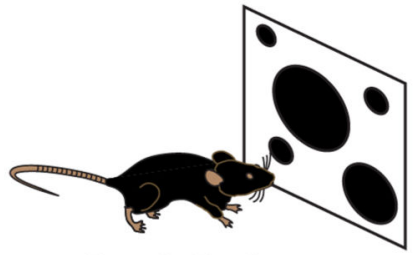

Day 2: Testing

Contextual fear conditioning

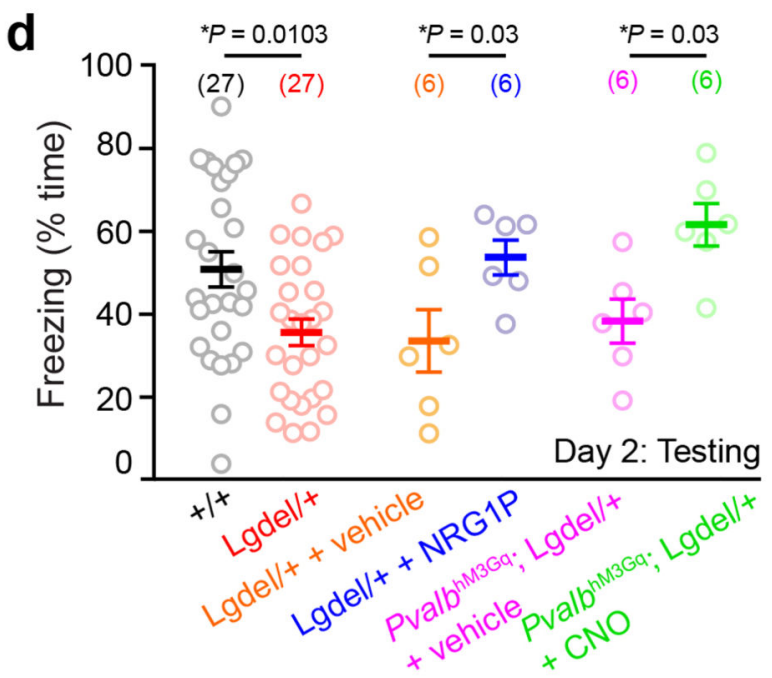

e

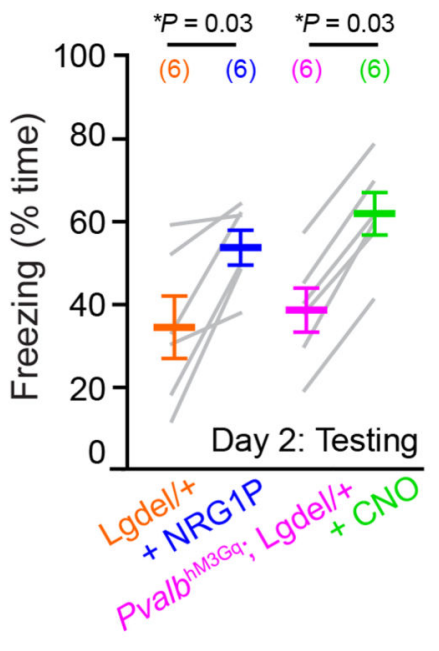

Fig. 7. Increasing PVI excitability during adulthood restores hippocampal-related behaviours. (a) Locomotor tracks of different transgenic mice and treatments during $10 \mathrm{~min}$ in an open field. (b) Summary graph of the distance travelled in the open field (two-sided MannWhitney test). (c) Schematic representation of the contextual fear conditioning procedure. (d) Summary graph of the freezing time for the different groups of mice (two-sided MannWhitney or two-sided Wilcoxon paired test). (e) Lgdel/+ mice freezing behaviour in the control conditions and after treatments with NRG1P (left) or CNO (right), respectively (twosided Wilcoxon paired test). All data are presented as mean \pm SEM. Each circle or gray line represents a mouse (total number of animals indicated in parentheses). 